\title{
Regularity-Constrained Pre- and Post-Filtering for Block DCT-Based Systems
}

\author{
Wei Dai, Student Member, IEEE, and Trac D. Tran, Member, IEEE
}

\begin{abstract}
It is well known that the traditional block transform can only have at most one degree of regularity. In other words, by retaining only one subband, these transforms, including the popular discrete cosine transform (DCT), can only capture the constant signal. The ability to capture polynomials of higher orders is critical in smooth signal approximation, minimizing blocking effects. This paper presents the theory, design, and fast implementation of regularity constrained pre-/post-filters for block-based decomposition systems. We demonstrate that simple pre-/post-filtering modules added to the current block-based infrastructure can help the block transform capture not only the constant signal but the ramp signal as well. Moreover, our proposed framework can be used to generate various fast symmetric $M$-band wavelets with up to two degrees of regularity.
\end{abstract}

Index Terms-DCT, $M$-band wavelets, linear-phase filterbanks, pre/post-filtering, pre-/post-processing, regularity, vanishing moments.

\section{INTRODUCTION}

$\mathbf{T}$ HE block-dicrete cosine transform (DCT) coding framework plays an important role in most image and video coding standards [1], [2]. In this coding framework, the input signal is partitioned into small local blocks of size $M$, each of which is decorrelated by the $M$-point DCT and then encoded by various quantization and entropy coding techniques. The block DCT coding framework does offer numerous advantages: fast-computable DCT algorithms; small on-board memory requirement; parallel processing capability; flexibility; and adaptability on the block level, e.g., coding mode can be selected on a block-by-block basis. Besides, there has been a tremendous number of software/hardware investments on block-based infrastructures over the last 20 years. It is a challenge to improve the coding efficiency as well as reconstruction quality, yet keep the existing infrastructures intact or minimize the required modifications.

Two major drawbacks of this block-based approach are that interblock correlation has been virtually ignored at the encoder, and reconstruction mismatch after aggressive quantization in low bit-rate situation creates annoying blocking artifacts at the decoder. Blocking artifacts are particularly prominent in smooth slowly changing regions of the signal. A typical example is that the staircase effect, where quantization of the AC of the DCT

Manuscript received December 17, 2001; revised February 24, 2003. The associate editor coordinating the review of this paper and approving it for publication was Dr. Helmut Boelcskei.

The authors are with the Department of Electrical and Computer Engineering, The Johns Hopkins University, Baltimore, MD 21218 USA (e-mail: wdai@jhu.edu; trac@jhu.edu).

Digital Object Identifier 10.1109/TSP.2003.816769 coefficients turns a ramp-like gradient signal such as a shading region in portrait photographs or a smooth blue sky in landscape photographs into a piece-wise constant signal. This problem stems from a lack of regularity - it is well known that the traditional block DCT can only have at most one degree of regularity. In other words, by retaining only DC coefficients, the transform can only capture the constant signal. The ability to capture polynomials of higher orders is critical in smooth signal approximation, alleviating blocking artifacts in signal reconstruction.

In this paper, we demonstrate that simple pre- and post-filtering modules added along the block boundaries of the current block DCT infrastructure can help the transform capture not only the constant signal but the ramp signal as well. The pre- and post-filtering framework is illustrated in Fig. 1 [3]. The pre-filter $\mathbf{P}$ processes the block boundaries, extracting interblock correlation. The pre-processed samples are then fed to the DCT to be transformed and encoded as usual. On the decoder side, $\mathbf{P}^{-1}$ serves as the post-filter, matching the signal from two sides of the block boundaries and eliminating blocking artifacts. Generally, $\left\{\mathbf{P}, \mathbf{P}^{-1}\right\}$ can be any invertible matrix pair. However, in most visual applications, linear phase is highly preferred. In other words, it is desirable to have symmetric/antisymmetric basis functions. Given the linear-phase DCT basis function, the particular proposed form of $\mathbf{P}$ not only guarantees symmetry but also reduces the design complexity. From Fig. 1, it is easy to see that the design of $\mathbf{P}$ boils down to the design of a smaller size matrix $\mathbf{V}$. Unlike the previous pre- and post-filtering approaches, the pre and post-filters here are intimately related. In fact, they are the exact inverse of each other, and together with the existing block DCT, they form a general block-based signal decomposition with arbitrary-length overlapping windows. Our focus in this paper is on the various properties and designs of the one-stage pre and post-filter depicted in Fig. 1(a) and (b). The proposed regularity-constrained pre and post-filters are also shown to be capable of generating various fast symmetric $M$-band wavelets with up to two vanishing moments and support up to length $2 M$.

The organization of the paper is as follows. In Section II, we offer a brief review of background materials: notations, conventions, pre-/post-filtering, the filterbank connection, regularity, vanishing moments, and $M$-band wavelets. Section III addresses the imposition of various regularity constraints onto the pre and post-filters to help the DCT capture polynomials more efficiently. Section IV introduces fast and efficient pre-/post-filter designs that have the regularity constraints in the Section III structurally built in. Numerous design examples are presented in Section V, and several interesting observations are discussed in Section VI. A simple image coding experiment 

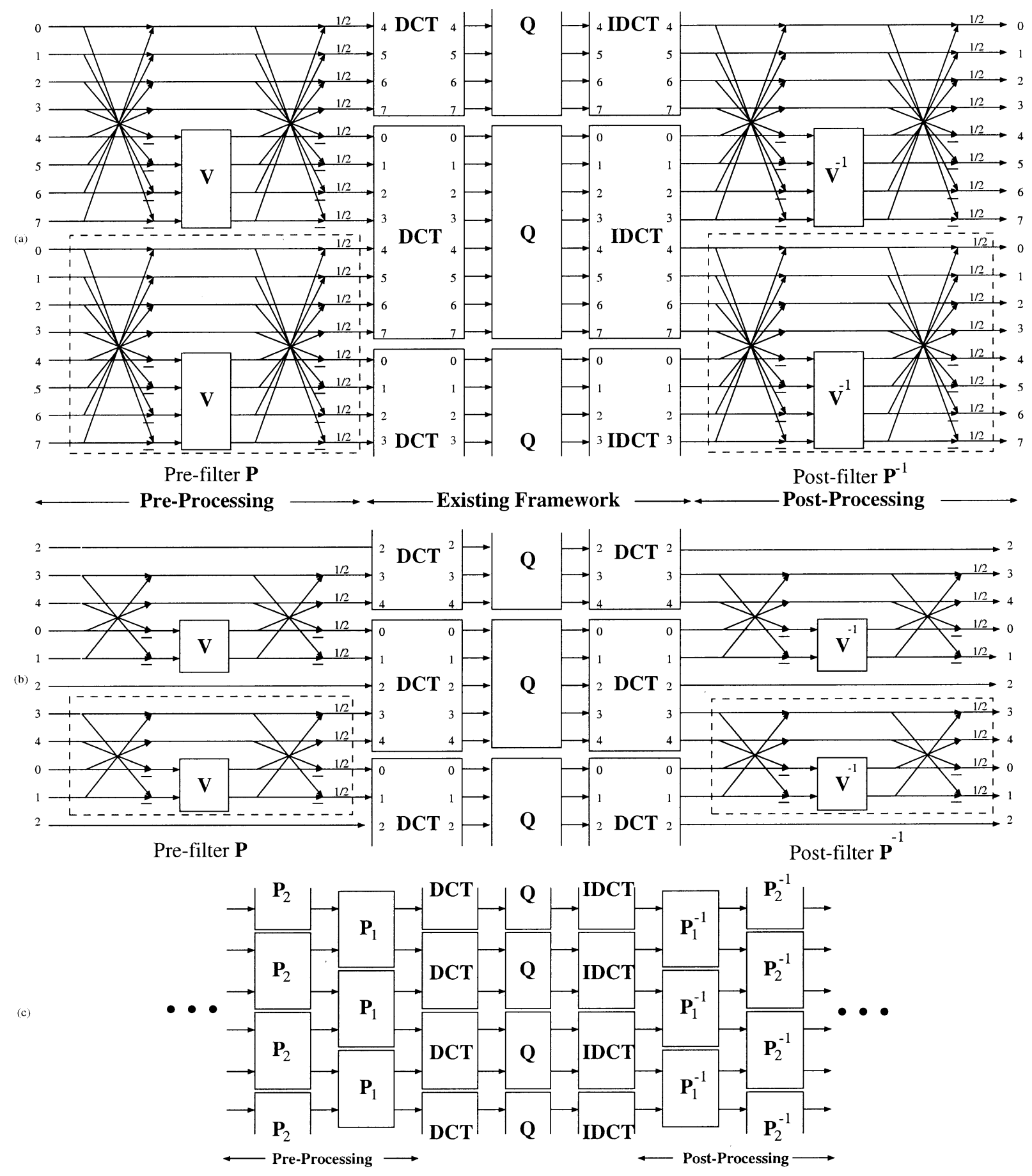

Fig. 1. Global viewpoint of pre-/post-filtering at block boundaries for (a) even block size; (b) odd block size; (c) general structure with multiple pre-/post-filtering stages.

is conducted in Section VII to confirm the validity of the theory, and final conclusions are drawn in Section VIII.

\section{BACKGROUND}

\section{A. Notations and Conventions}

Bold-faced upper-case characters denote matrices, while bold-faced lower-case characters denote vectors. The symbols $\mathbf{A}^{T}$ and $\mathbf{A}^{-1}$ denote the transpose and the inverse of the matrix $\mathbf{A}$, respectively. Several reserved symbols are the $M$-point type-II DCT matrix $\mathrm{C}_{M}^{I I}$, the $M$-point type-IV DCT matrix $\mathbf{C}_{M}^{I V}$, the $M$-point type-II IDCT matrix $\mathbf{C}_{M}^{I I^{T}}$, the
$M$-point type-IV IDCT matrix $\mathbf{C}_{M}^{I V^{T}}$, the identity matrix $\mathbf{I}$, the reversal matrix $\mathbf{J}$ (I flipped left-right), the null matrix $\mathbf{0}$, the column unity vector $\mathbf{u}=\left[\begin{array}{llll}1 & 1 & \cdots & 1\end{array}\right]^{T}$, the ramp vector of size $N$ with slope $1: \mathbf{r}=\left[\begin{array}{llll}1 & 2 & \cdots & N\end{array}\right]^{T}$, the ramp vector of size $N$ with odd-valued samples and slope $2: \mathbf{q}=\left[\begin{array}{llll}1 & 3 & \cdots & 2 N-1\end{array}\right]^{T}$, and the column vector with all entries being zero, except the first entry being unity $\mathbf{e}=\left[\begin{array}{llll}1 & 0 & \cdots & 0\end{array}\right]^{T}$. We denote the flooring function of $x$ as $\lfloor x\rfloor$, i.e., the largest integer less than or equal to $x$.

In most cases, the size of matrices and vectors, i.e., $\mathbf{u}, \mathbf{r}, \mathbf{q}, \mathbf{e}$ can be readily determined from the context. If it is not clear from 
(a)
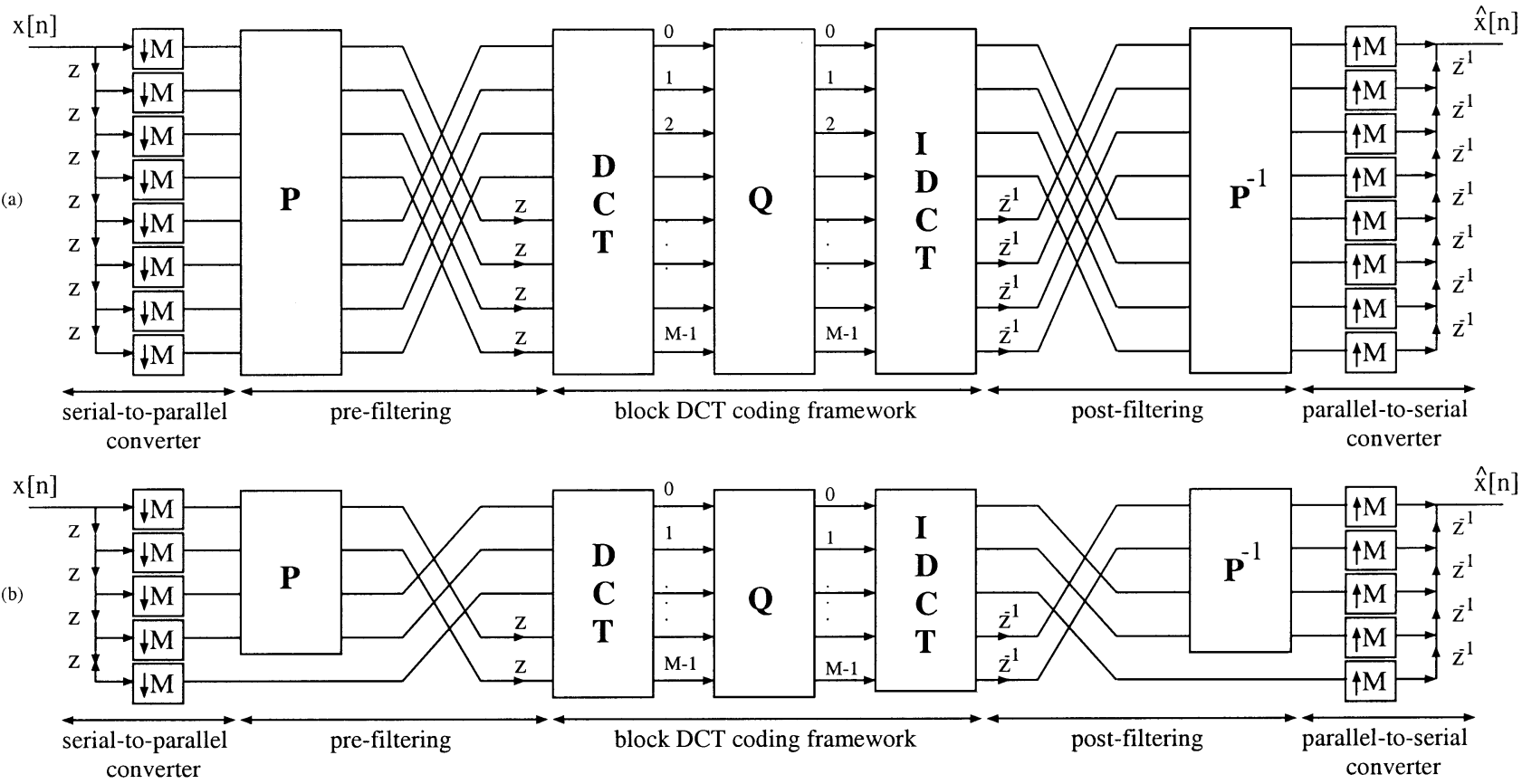

Fig. 2. Polyphase representation of the pre-/post-filtering framework. (a) Even block size. (b) Odd block size.

the context, uppercase subscripts are added to indicate the size of the matrices or vectors. Lower-case subscripts are reserved for indexing purpose.

\section{B. Filterbank Connection}

An equivalent representation of the pre-/post-filtering framework in Fig. 1 is shown in Fig. 2, which can be interpreted as the polyphase implementation of an $M$-band uniform filterbank. The pre-filtering operator $\mathbf{P}$ consists of two stages of butterflies and a matrix $\mathbf{V}$ between them

$$
\mathbf{P} \triangleq \frac{1}{2}\left[\begin{array}{cc}
\mathbf{I} & \mathbf{J} \\
\mathbf{J} & -\mathbf{I}
\end{array}\right]\left[\begin{array}{cc}
\mathbf{I} & \mathbf{0} \\
\mathbf{0} & \mathbf{V}
\end{array}\right]\left[\begin{array}{cc}
\mathbf{I} & \mathbf{J} \\
\mathbf{J} & -\mathbf{I}
\end{array}\right] .
$$

The corresponding polyphase matrix for even block size is

$$
\begin{aligned}
\mathbf{E}_{\text {even }}(z) \triangleq & \mathbf{C}_{M}^{I I} \boldsymbol{\Lambda}_{\text {even }}(z) \mathbf{P} \\
= & \mathbf{C}_{M}^{I I}\left[\begin{array}{cc}
\mathbf{0} & \mathbf{I} \\
z \mathbf{I} & \mathbf{0}
\end{array}\right] \\
& \times \frac{1}{2}\left[\begin{array}{cc}
\mathbf{I} & \mathbf{J} \\
\mathbf{J} & \mathbf{- I}
\end{array}\right]\left[\begin{array}{cc}
\mathbf{I} & \mathbf{0} \\
\mathbf{0} & \mathbf{V}
\end{array}\right]\left[\begin{array}{cc}
\mathbf{I} & \mathbf{J} \\
\mathbf{J} & \mathbf{- I}
\end{array}\right]
\end{aligned}
$$

whereas the polyphase matrix for odd block size is

$$
\begin{aligned}
\mathbf{E}_{\text {odd }}(z) \triangleq & \mathbf{C}_{M}^{I I} \boldsymbol{\Lambda}_{\text {odd }}(z) \mathbf{P}^{\prime} \\
= & \mathbf{C}_{M}^{I I}\left[\begin{array}{cc}
\mathbf{0}_{((M+1) / 2) \times((M-1) / 2)} & \mathbf{I}_{(M+1) / 2} \\
z \mathbf{I}_{(M-1) / 2} & \mathbf{0}_{((M-1) / 2) \times((M+1) / 2)}
\end{array}\right] \\
& \times\left[\begin{array}{cc}
\mathbf{P}_{(M-1)} & \mathbf{0}_{(M-1) \times 1} \\
\mathbf{0}_{1 \times(M-1)} & 1
\end{array}\right]
\end{aligned}
$$

Here

$$
\boldsymbol{\Lambda}_{\mathrm{even}}(z) \triangleq\left[\begin{array}{cc}
\mathbf{0} & \mathbf{I} \\
z \mathbf{I} & \mathbf{0}
\end{array}\right]
$$

and

$$
\boldsymbol{\Lambda}_{\text {odd }}(z) \triangleq\left[\begin{array}{cc}
\mathbf{0}_{((M+1) / 2) \times((M-1) / 2)} & \mathbf{I}_{(M+1) / 2} \\
z \mathbf{I}_{(M-1) / 2} & \mathbf{0}_{((M-1) / 2) \times((M+1) / 2)}
\end{array}\right]
$$

are the permuted advanced chains, and they play the central role in the time-domain pre-/post-processing across block boundaries. It can be easily verified that the determinant of the polyphase matrix $\mathbf{E}(z)$ in (2) and (3) is a monomial, i.e., $|\mathbf{E}(z)|=z^{n}, n \in \mathcal{Z}$; hence, one can obtain FIR perfect reconstruction by simply choosing $\mathbf{R}(z)=\mathbf{E}^{-1}(z)$ [4]-[6]. The $z$-transforms of the analysis filters and synthesis filters in matrix notation are

$$
\begin{aligned}
& {\left[\begin{array}{llll}
H_{0}(z) & H_{1}(z) & \cdots & H_{M-1}(z)
\end{array}\right]^{T} } \\
= & \mathbf{E}\left(z^{M}\right)\left[\begin{array}{cccc}
1 & z & \cdots & z^{M-1}
\end{array}\right]^{T} \\
& {\left[\begin{array}{lllll}
F_{0}(z) & F_{1}(z) & \cdots & F_{M-1}(z)
\end{array}\right] } \\
= & {\left[\begin{array}{lllll}
1 & z^{-1} & \cdots & z^{-(M-1)}
\end{array}\right] \mathbf{R}\left(z^{M}\right) }
\end{aligned}
$$

respectively. The structure of the pre-filter $\mathbf{P}$ in (1) ensures that all analysis and synthesis filters have linear phase, which is a desirable feature in image and video processing applications.

Since every component in $\mathbf{E}(z)$ is paraunitary except the free parameter matrix $\mathbf{V}$, if $\mathbf{V}$ is chosen orthogonal $\left(\mathbf{V}^{-1}=\mathbf{V}^{T}\right)$, we have an orthogonal solution. In this case, $\mathbf{P}^{-1}=\mathbf{P}^{T}$ and $\mathbf{R}(z)=\mathbf{E}^{T}\left(z^{-1}\right)$. Otherwise, if $\mathbf{V}$ is only invertible, we have a biorthogonal solution. The matrix $\mathbf{V}$ holds all of the degrees of freedom in this structure, thus controlling the performance and properties of pre-/post-filtering.

At first glance, it seems that the framework in Fig. 1(a) can only generate filters $\left\{H_{i}(z), F_{i}(z)\right\}$ of length $2 M$, whereas the framework in Fig. 1(b) can only generate filters of length (2M1). However, by controlling $\mathbf{V}$ properly, we can also obtain filters of shorter support. For example, if $\mathbf{V}=\mathbf{I}$, then $\mathbf{P}=\mathbf{I}$, and the entire structure degenerates to the familiar block-based DCT. If $\mathbf{V}$ takes the form of

$$
\mathbf{V}=\left[\begin{array}{cc}
\hat{\mathbf{V}}_{N \times N} & \mathbf{0}_{N \times\lfloor(M-2 N) / 2\rfloor} \\
\mathbf{0}_{\lfloor(M-2 N) / 2\rfloor \times N} & \mathbf{I}_{\lfloor(M-2 N) / 2\rfloor}
\end{array}\right]
$$


then the resulting filter length is $M+2 N$, where $0 \leq N \leq$ $\lfloor M / 2\rfloor$. To obtain filters with longer support, more pre-/postfiltering stages are required. Each processing stage works at the boundary of the previous stage as illustrated in Fig. 1(c). The same symmetry constraint as in (1) can be added to the building block $\mathbf{P}_{i}$ if linear phase filters are desired. The matrices $\mathbf{V}_{i}$ 's in different $\mathbf{P}_{i}$ stages are not necessarily the same. They can be optimized to achieve optimal performance for specific applications.

\section{Review of Regularity}

A recursive cascade of the previously mentioned $M$-band decomposition on the lowpass channel output can generate $M$-band wavelets. Smoothness of the resulting continuous-time scaling function $\phi(t)$ and the wavelet function $\psi(t)$ is crucial in signal approximation and interpolation. In this section, we shall follow the wavelet community's definition of regularity, which is a loose measure of the smoothness of the scaling filter. We also review several important results regarding the regularity of $M$-band orthogonal and biorthogonal filterbanks. First, the Fourier transforms of the analysis and synthesis scaling and wavelet functions $\phi(t)$ and $\psi_{l}(t)$, for $l=1,2, \ldots, M-1$, are defined as

$$
\begin{aligned}
\Phi_{a}(\omega) & =\prod_{k=1}^{\infty}\left[\frac{1}{\sqrt{M}} H_{0}\left(\frac{\omega}{M^{k}}\right)\right] \\
\Phi_{s}(\omega) & =\prod_{k=1}^{\infty}\left[\frac{1}{\sqrt{M}} F_{0}\left(\frac{\omega}{M^{k}}\right)\right] \\
\Psi_{a, l}(\omega) & =\frac{1}{\sqrt{M}} H_{l}\left(\frac{\omega}{M}\right) \prod_{k=2}^{\infty}\left[\frac{1}{\sqrt{M}} H_{0}\left(\frac{\omega}{M^{k}}\right)\right] \\
\Psi_{s, l}(\omega) & =\frac{1}{\sqrt{M}} F_{l}\left(\frac{\omega}{M}\right) \prod_{k=2}^{\infty}\left[\frac{1}{\sqrt{M}} F_{0}\left(\frac{\omega}{M^{k}}\right)\right] .
\end{aligned}
$$

Definition 1: The lowpass filter $H_{0}(z)$ is said to be a $K$-regular $M$-band scaling filter if it can be written in the form of $H_{0}(z)=\left[\left(1+z^{-1}+\cdots+z^{-(M-1) / M}\right)\right]^{K} Q(z)$ [7].

Definition 2: An $M$-band biorthogonal filterbank is said to be $\left\{K_{a}, K_{s}\right\}$-regular if the analysis lowpass filter $H_{0}(z)$ is $K_{a}$-regular and the synthesis lowpass filter $F_{0}(z)$ is $K_{s}$-regular [8].

Theorem 1: (Equivalent Characterizations of K-Regular Orthogonal $M$-Band Filters) [7], [9]: In an $M$-band orthogonal filterbank, the scaling filter is $K$-regular, has $K$ degree of regularity, or has $K$ vanishing moments if and only if the following equivalent statements are true.

1) All moments up to order $K-1$ of the $M-1$ wavelet filters are zeros, i.e., $\sum_{n} n^{k} h_{i}[n]=0$, for $k=0,1, \ldots,(K-$ 1) and for $i=1,2, \ldots, M-1$.

2) The frequency response of the scaling filter has a zero of order $K$ at the $M$ th root of unity $\omega=2 k \pi / M$ for $k=1,2, \ldots, M-1$.

3) All polynomial sequences up to degree $(K-1)$ can be expressed as a linear combination of $M$-integer-shifted scaling filters. All polynomials of degree up to $(K-1)$ can be expressed as a linear combination of integer-shifted scaling functions.
Theorem 2: (Equivalent Characterizations of $\left\{K_{a}, K_{s}\right\}$-Regular Biorthogonal $M$-Band Filters) [8]: In an $M$-band biorthogonal filterbank, the analysis scaling filter $H_{0}(z)$ is $K_{a}$-regular, and the synthesis scaling filter $F_{0}(z)$ is $K_{s}$-regular if and only if the following equivalent statements are true.

1) All moments up to order $K_{s}-1$ of the $M-1$ analysis wavelet filters are zeros, i.e., $\sum_{n} n^{k} h_{i}[n]=0$, for $k=$ $0,1, \ldots,\left(K_{s}-1\right)$ and for $i=1,2, \ldots,(M-1)$. All moments up to order $K_{a}-1$ of the $M-1$ synthesis wavelet filters are zeros, i.e., $\sum_{n} n^{k} f_{i}[n]=0$, for $k=$ $0,1, \ldots,\left(K_{a}-1\right)$ and for $i=1,2, \ldots, M-1$.

2) The frequency response of the analysis scaling filter has a zero of order $K_{a}$ at the $M$ th root of unity $\omega=2 k \pi / M$ for $k=1,2, \ldots, M-1$. The frequency response of the synthesis scaling filter has a zero of order $K_{s}$ at the $M$ th root of unity $\omega=2 k \pi / M$ for $k=1,2, \ldots, M-1$.

3) All polynomial sequences up to degree $\left(K_{a}-1\right)$ can be expressed as a linear combination of $M$-integer-shifted analysis scaling filters. On the other hand, all polynomial sequences up to degree $K_{s}-1$ can be expressed as a linear combination of $M$-integer-shifted synthesis scaling filters.

The concept of polynomial sequence capturing and representation is the direct method we pursue when enforcing more vanishing moments on the synthesis filters.

\section{Regularity CONSTRAints FOR PRE/Post-FILTERING}

As previously mentioned, the filterbank generated by the one stage of pre-/post-filtering in Fig. 2 has a filter length up to $2 M$. According to the Definition 1, a $K$-regular $M$-band scaling filter has the form $H_{0}(z)=\left[\left(1+z^{-1}+\cdots+z^{-(M-1)} / M\right)\right]^{K} Q(z)$. The filter length $L$ should satisfy $L \geq K(M-1)+1$ with equality when $Q(z)$ is a constant. Thus, $K \leq L-1 /(M-1) \leq$ $(2 M-1) /(M-1) \leq 2+1 /(M-1)$. When $M=2$, a 3-regular 2-bank 4-tap filter bank is a possibility. However, when $M>2$ and $L \leq 2 M$, we can only expect to have at most two vanishing moments, implying three possibilities: $\{1,2\}$-regularity, $\{2,1\}$-regularity, and $\{2,2\}$-regularity. This can be summarized as Proposition 1.

Proposition 1: An $M$-channel $2 M$-tap filterbank has a maximum number of two vanishing moments when $M>2$.

If higher degrees of regularity are desired, we need to cascade more pre- and post-filtering stages, as shown in Fig. 1(c).

Consider the block DCT coding framework in Fig. 2. Let $M$ denote the DCT block size. Notice that the $M$-point DCT is a 1-regular orthogonal $M$-band $M$-tap filterbank with unitary scaling filter $H_{0}(z)=F_{0}(z)=$ $\left(1+z^{-1}+\cdots+z^{-(M-1))} / \sqrt{M}\right.$ (here, $Q(z)$ is chosen as $\sqrt{M})$. The original problem statement is simplified to the following: Design the pre-filter $\mathbf{P}$ and the post-filter $\mathbf{P}^{-1}$ such that the resulting combined decomposition obtains the maximum possible number of vanishing moments $\left\{K_{a}, K_{s}\right\}$. In other words, given the input as a polynomial sequence of a particular order, design $\mathbf{P}$ and $\mathbf{P}^{-1}$ such that the DC coefficients of the DCT capture all of the input energy. For this purpose, the first equivalent characterization in Theorems 1 and 
2 turn out to be both intuitive and effective. The characterization can be interpreted as polynomial capturing and polynomial rejection. Since $\sum_{n} n^{k} h_{i}[n]=0$ for $i=1,2, \ldots,(M-1)$, the polynomial input is rejected by all AC coefficients, and it can be solely captured in the DC coefficients. Since the matrix V contains all free parameters of pre-/post-filtering, we expect to be able to express every regularity condition in term of this crucial matrix.

\section{A. 1-Regular Condition}

For convenience, denote $\mathbf{x}_{m}$ as the input sequence to each pre-filter $\mathbf{P}, \hat{\mathbf{x}}_{m}$ as the resulting preprocessed sequence, and $\mathbf{y}_{m}$ as the input to each DCT block.

Lemma 1: The pre-/post-filter produces a $K_{a}\left(K_{s}\right)$-regular, $K_{a}, K_{s} \geq 1$, analysis (synthesis) scaling filter if and only if it can map all polynomial sequences up to degree $K_{s}-1\left(K_{a}-1\right)$ into a piece-wise constant signal, or, equivalently, the input to each block DCT is constant.

Proof: According to Theorem 2, all polynomial sequences up to degree $K_{s}-1$ can be captured by the analysis scaling filter and rejected by the analysis wavelet filters. From Fig. 1, this means that the output of each block DCT is zero, except the DC coefficient. Since the $M$-point DCT has one vanishing moment, it can capture only the constant sequence; thus, the block input to the $M$-point forward DCT should be a piece-wise constant sequence.

By changing roles of $\left(\mathbf{P}, \mathbf{P}^{-1}\right)$ and $\left(K_{a}, K_{s}\right)$, we can obtain the same result for the synthesis bank.

Lemma 2: The pre-filter $\mathbf{P}$ is a mirror mapping operator for constant sequences, i.e., $\mathbf{P u}=\mathbf{u}$.

Proof:

$$
\begin{aligned}
\mathbf{P u} & =\frac{1}{2}\left[\begin{array}{cc}
\mathbf{I} & \mathbf{J} \\
\mathbf{J} & -\mathbf{I}
\end{array}\right]\left[\begin{array}{cc}
\mathbf{I} & \mathbf{0} \\
\mathbf{0} & \mathbf{V}
\end{array}\right]\left[\begin{array}{cc}
\mathbf{I} & \mathbf{J} \\
\mathbf{J} & -\mathbf{I}
\end{array}\right] \mathbf{u} \\
& =\left[\begin{array}{cc}
\mathbf{I} & \mathbf{J V} \\
\mathbf{J} & -\mathbf{V}
\end{array}\right]\left[\begin{array}{l}
\mathbf{u}_{M / 2} \\
\mathbf{0}_{M / 2}
\end{array}\right]=\mathbf{u} .
\end{aligned}
$$

Note that Lemma 2 holds, regardless of the choice of $\mathbf{V}$. This result is quite intuitive from Fig. 1(a) and (b). When the input is a constant sequence, after the butterfly, the differences of the input are zeros, rendering $\mathbf{V}$ insignificant. As long as the pre-filter has this particular structure, the mirror property of $\mathbf{P}$ does not depend on $\mathbf{V}$. Next, since the post-filter $\mathbf{P}^{-1}$ also has the same structure, we have $\mathbf{P}^{-1} \mathbf{u}=\mathbf{u}$ as well as $\mathbf{P}^{-T} \mathbf{u}=\mathbf{u}$. Lemmas 1 and 2 readily establish the following 1-regular condition.

Theorem 3: The pre-/post-filter always produces analysis (synthesis) scaling filter with at least one vanishing moment, regardless of the choice of $\mathbf{V}$.

Proof: Refer to Fig. 1(a)-(b). Suppose that the input $\mathbf{x}$ is a constant sequence. Since the pre-filter $\mathbf{P}$ is a mirror operator according to Lemma 2, the input to the block DCT is also a constant sequence. From the result established in Lemma 1, the scaling filter has at least one vanishing moment.

\section{B. 2-Regular Condition}

In this paper, we only discuss filterbanks produced by one stage of pre-/post-filtering. Therefore, the scaling filter can only take the following forms: $H_{0}(z)=$ $\left[\left(1+z^{-1}+\cdots+z^{-(M-1)} / M\right)\right]^{2}\left(a+a z^{-1}\right)$ for $M$ even and $H_{0}(z)=a\left[\left(1+z^{-1}+\cdots+z^{-(M-1)} / M\right)\right]^{2}$ for $M$ odd.

Theorem 4: The pre-filter $\mathbf{P}$ produces a 2-regular synthesis scaling filter if and only if $\mathbf{V q}=M \mathbf{u}$. Analogously, the postfilter $\mathbf{P}^{-1}$ produces a 2-regular analysis scaling filter if and only if $\mathbf{V}^{-T} \mathbf{q}=M \mathbf{u}$.

Proof:

a) When $M$ is even, the size of the pre-filter $\mathbf{P}$ is $M \times M$, and the size of $\mathbf{V}$ is $M / 2 \times M / 2$. Consider two connected input sequences $\left\{\mathbf{x}_{m}, \mathbf{x}_{m+1}\right\}$, which form a continuous $\operatorname{ramp} \mathbf{x}_{m}=\left[\begin{array}{llll}1 & 2 & \ldots & M\end{array}\right]^{T}=\mathbf{r}_{M}$ and $\mathbf{x}_{m+1}=$ $\left[\begin{array}{llll}M+1 & M+2 & \ldots & 2 M\end{array}\right]^{T}=M \mathbf{u}_{M}+\mathbf{r}_{M}$. The signals after preprocessing are $\hat{\mathbf{x}}_{m}=\mathbf{P} \mathbf{x}_{m}$ and $\hat{\mathbf{x}}_{m+1}=$ $\mathbf{P x}_{m+1}$, respectively

$$
\begin{aligned}
\hat{\mathbf{x}}_{m} & =\frac{1}{2}\left[\begin{array}{cc}
\mathbf{I} & \mathbf{J} \\
\mathbf{J} & -\mathbf{I}
\end{array}\right]\left[\begin{array}{cc}
\mathbf{I} & \mathbf{0} \\
\mathbf{0} & \mathbf{V}
\end{array}\right]\left[\begin{array}{cc}
\mathbf{I} & \mathbf{J} \\
\mathbf{J} & -\mathbf{I}
\end{array}\right] \mathbf{r}_{M} \\
& =\frac{1}{2}\left[\begin{array}{cc}
\mathbf{I} & \mathbf{J V} \\
\mathbf{J} & -\mathbf{V}
\end{array}\right]\left[\begin{array}{c}
(M+1) \mathbf{u} \\
-\mathbf{q}
\end{array}\right] \\
& =\frac{1}{2}\left[\begin{array}{c}
(M+1) \mathbf{u}-\mathbf{J V q} \\
(M+1) \mathbf{u}+\mathbf{V q}
\end{array}\right], \\
\hat{\mathbf{x}}_{m+1} & =\mathbf{P}\left(M \mathbf{u}_{M}+\mathbf{r}_{M}=M \mathbf{u}_{M}+\mathbf{P r}_{M}\right. \\
& =M \mathbf{u}_{M}+\frac{1}{2}\left[\begin{array}{c}
(M+1) \mathbf{u}-\mathbf{J V q} \\
(M+1) \mathbf{u}+\mathbf{V q}
\end{array}\right] .
\end{aligned}
$$

Therefore, the input to the DCT is

$$
\begin{aligned}
& \mathbf{y}_{m}=\left[\begin{array}{llll}
\mathbf{0}_{M / 2} & \mathbf{I}_{M / 2} & \mathbf{0}_{M / 2} & \mathbf{0}_{M / 2} \\
\mathbf{0}_{M / 2} & \mathbf{0}_{M / 2} & \mathbf{I}_{M / 2} & \mathbf{0}_{M / 2}
\end{array}\right]\left[\begin{array}{c}
\hat{\mathbf{x}}_{m} \\
\hat{\mathbf{x}}_{m+1}
\end{array}\right] \\
& =\frac{1}{2}\left[\begin{array}{c}
(M+1) \mathbf{u}+\mathbf{V q} \\
(3 M+1) \mathbf{u}-\mathbf{J V q}
\end{array}\right] .
\end{aligned}
$$

The input signal can be completely captured by the lowpass subband or the DC coefficient if and only if $\mathbf{y}_{m}$ is a constant sequence, implying that $\mathbf{V q}$ must be a constant signal, i.e., $\mathbf{V q}=S \mathbf{u}$, where $S$ is a certain scalar. Thus, $(M+1) \mathbf{u}+\mathbf{V q}=(3 M+1) \mathbf{u}-\mathbf{J V q} \Longleftrightarrow$ $(M+1+S) \mathbf{u}=(3 M+1-S) \mathbf{u} \Longleftrightarrow S=M$, and $\mathbf{V q}=M \mathbf{u}$.

b) When $M$ is odd, the input sequences to the pre-filter $\mathbf{P}$ are $\mathbf{x}_{m}=\left[\begin{array}{llll}1 & 2 & \cdots & M-1\end{array}\right]^{T}=\mathbf{r}_{M-1}, \mathbf{x}_{m+1}=$ $\left[\begin{array}{llll}M+1 & M+2 & \cdots & 2 M-1\end{array}\right]^{T}=M \mathbf{u}+\mathbf{r}_{M-1}$, $\mathbf{x}_{m+2}=\left[\begin{array}{llll}2 M+1 & 2 M+2 & \cdots & 3 M-1\end{array}\right]^{T}=$ $2 M \mathbf{u}+\mathbf{r}_{M-1}$, etc. The size of the pre-filter $\mathbf{P}$ is $(M-1) \times(M-1)$, and the size of $\mathbf{V}$ is $(M-1) / 2 \times(M-1) / 2$.

The outputs of pre-filter $\mathbf{P}$ are

$$
\begin{aligned}
\hat{\mathbf{x}}_{m} & =\frac{1}{2}\left[\begin{array}{cc}
\mathbf{I} & \mathbf{J} \\
\mathbf{J} & -\mathbf{I}
\end{array}\right]\left[\begin{array}{cc}
\mathbf{I} & \mathbf{0} \\
\mathbf{0} & \mathbf{V}
\end{array}\right]\left[\begin{array}{cc}
\mathbf{I} & \mathbf{J} \\
\mathbf{J} & -\mathbf{I}
\end{array}\right] \mathbf{r}_{M-1} \\
& =\frac{1}{2}\left[\begin{array}{cc}
\mathbf{I} & \mathbf{J V} \\
\mathbf{J} & -\mathbf{V}
\end{array}\right]\left[\begin{array}{c}
M \mathbf{u} \\
-\mathbf{q}
\end{array}\right]=\frac{1}{2}\left[\begin{array}{c}
M \mathbf{u}-\mathbf{J V q} \\
M \mathbf{u}+\mathbf{V q}
\end{array}\right] \\
\hat{\mathbf{x}}_{m+1} & =\mathbf{P}\left(M \mathbf{u}_{M-1}+\mathbf{r}_{M-1}\right)=M \mathbf{u}_{M-1}+\mathbf{P r}_{M-1} \\
& =M \mathbf{u}_{M-1}+\frac{1}{2}\left[\begin{array}{c}
M \mathbf{u}-\mathbf{J V q} \\
M \mathbf{u}+\mathbf{V q}
\end{array}\right] .
\end{aligned}
$$

The input to the DCT is shown in the equation at the bottom of the next page. The synthesis filter is 2-regular if and only if $\mathbf{y}_{m}$ is a constant sequence, that is, $\mathbf{M u}+\mathbf{V q}=$ $2 M \mathbf{u}=3 M \mathbf{u}-\mathbf{J V q} \Longleftrightarrow \mathbf{V q}=M \mathbf{u}$. 
c) Switching the role of the analysis and synthesis bank, we can easily establish the analogous result for a 2-regular analysis filter: $\mathbf{V}^{-T} \mathbf{q}=M \mathbf{u}$.

\section{Orthogonal Solution}

Recall that we have orthogonal pre-/post-filter if the $\lfloor M / 2\rfloor \times$ $\lfloor M / 2\rfloor$ matrix $\mathbf{V}$ is orthogonal, i.e., $\mathbf{V}^{-1}=\mathbf{V}^{T}$. In this case, the analysis and synthesis scaling filters will have the same numbers of vanishing moments. Due to the strict orthogonal constraint, we can only obtain at most a $\{1,1\}$-regular orthogonal $M$-band system with one stage of pre-/post-filtering.

Theorem 5: The pre-filter $\mathbf{P}$ and the post-filter $\mathbf{P}^{-1}$ can only produce an orthogonal $M$-band system with at most one vanishing moment.

Proof: Suppose that there exists an $M$-band orthogonal filterbank produced by $\mathbf{P}$ and $\mathbf{P}^{-1}$ with two vanishing moments. Then, according to Theorem 4 , the orthogonal matrix $\mathbf{V}$ must satisfy the regularity constraint $\mathbf{V q}=M \mathbf{u}$, or, equivalently, $\mathbf{q}^{T} \mathbf{V}^{T}=M \mathbf{u}^{T}$. Of course, $M$ is a positive integer larger than unity, i.e., $M \geq 2$. In this case

$$
\begin{aligned}
\mathbf{q}^{T} \mathbf{V}^{T} \mathbf{V} \mathbf{q} & =M^{2} \mathbf{u}^{T} \mathbf{u} \Longrightarrow \mathbf{q}^{T} \mathbf{q}=M^{2}\left\lfloor\frac{M}{2}\right\rfloor \\
& \Longrightarrow \sum_{i=1}^{\lfloor M / 2\rfloor}(2 i-1)^{2}=M^{2}\left\lfloor\frac{M}{2}\right\rfloor \\
& \Longrightarrow \frac{1}{3}\left\lfloor\frac{M}{2}\right\rfloor\left(4\left\lfloor\frac{M}{2}\right\rfloor^{2}-1\right)=M^{2}\left\lfloor\frac{M}{2}\right\rfloor
\end{aligned}
$$

On the other hand, we also have

$$
\begin{aligned}
\frac{1}{3}\left\lfloor\frac{M}{2}\right\rfloor\left(4\left\lfloor\frac{M}{2}\right\rfloor^{2}-1\right) & \leq \frac{M}{6}\left(M^{2}-1\right)=\frac{M+1}{3} \frac{M(M-1)}{2} \\
& <M \frac{M(M-1)}{2} \leq M^{2}\left\lfloor\frac{M}{2}\right\rfloor
\end{aligned}
$$

which is contradictory to (4). Therefore, a matrix $\mathbf{V}$ that can produce a 2-regular orthogonal $M$-band system does not exist.

\section{Biorthogonal Solution}

In a biorthogonal filterbank, the number of analysis and synthesis vanishing moments can be different. In this section, we will show that although a $\{1,2\}$-regular or a $\{2,1\}$-regular system is possible, it is still impossible to design a $\{2,2\}$-regular biorthogonal $M$-band wavelet with only one stage of
TABLE I

SUMMARY OF THE NECESSARY AND SUFFICIENT CONDITIONS FOR REGULAR $M$-BANd Filterbanks ProducEd By PRE/POSTFILTERING

\begin{tabular}{c||c|c}
\hline Regularity & Orthogonal & Biorthogonal \\
\hline$\{1,1\}$ & any orthogonal V & any invertible V \\
$\{1,2\}$ & impossible & $\mathbf{V q}=M \mathbf{u}$ \\
$\{2,1\}$ & impossible & $\mathbf{V}^{-T} \mathbf{q}=M \mathbf{u}$ \\
$\{2,2\}$ & impossible & impossible \\
$\{1,3\}$ & impossible & $\mathbf{V}=2, M=2$ \\
$\{3,1\}$ & impossible & $\mathbf{V}=\frac{1}{2}, M=2$ \\
\hline
\end{tabular}

pre-/post-filtering. This can be explained in a similar fashion as in the orthogonal case.

Theorem 6: It is impossible for the pre-filter $\mathbf{P}$ and the postfilter $\mathbf{P}^{-1}$ to produce a biorthogonal $\{2,2\}$-regular $M$-band system.

Proof: Since we need to impose two vanishing moments on both banks, a nonsingular matrix $\mathbf{V}$ should satisfy both of the following requirements: $\mathbf{V q}=M \mathbf{u}$ and $\mathbf{V}^{-T} \mathbf{q}=M \mathbf{u}$. Similarly, we obtain the same contradiction when the DCT block size $M \geq 2: \mathbf{q}^{T} \mathbf{V}^{-1} \mathbf{V q}=\mathbf{q}^{T} \mathbf{q}=M^{2} \mathbf{u}^{T} \mathbf{u}=M^{2}\lfloor M / 2\rfloor$.

To summarize, the $\{1,1\}$-regular condition is automatically satisfied for any invertible matrix $\mathbf{V}$. The $\{1,2\}-$ or $\{2,1\}$-regular condition is possible if and only if the regularity constraint $\mathbf{V q}=\mathbf{u}$ or $\mathbf{V}^{-T} \mathbf{q}=\mathbf{u}$ is satisfied, respectively. The $\{2,2\}$-regular condition is not achievable. When $M=2$, we have the only trivial $\{1,3\}$ or $\{3,1\}$-regular system with $\mathbf{V}=[2]$ or $\mathbf{V}=[1 / 2]$. These results are tabulated in Table I. In practice, we expect smoother basis functions in the synthesis bank. Therefore, in general, we prefer $\{1,2\}$-regularity over $\{2,1\}$-regularity.

\section{FAST IMPLEMENTATIONS OF REGULAR PRE- AND POST-FILTER}

The free-parameter matrix $\mathbf{V}$ can be modeled using the LDU decomposition. The upper triangular matrix $\mathbf{U}$ and the lower triangular matrix $\mathbf{L}$ are constructed by cascading lifting steps. However, modeling the matrix $\mathbf{V}$ as described above leads to a computational overhead in the order of $\mathcal{O}\left(M^{2}\right)$ multiplications and additions comparing to the complexity of the DCT without pre-/post-processing. To further reduce the complexity of the pre- and post-filter, several simplified models for $\mathbf{V}$ providing significant computational saving while maintaining coding performance have been proposed [10]. Two of such models are depicted in Fig. 3. Following [10], we label the corresponding preprocessing operators the type-III and the type-IV pre-filter, respectively. Note that when $M \leq 4$, the type-III and -IV prefilter are equivalent.

$$
\begin{aligned}
\mathbf{y}_{m} & =\left[\begin{array}{ccccc}
\mathbf{0}_{(M-1) / 2} & \mathbf{I}_{(M-1) / 2} & \mathbf{0}_{(M-1) / 2 \times 1} & \mathbf{0}_{(M-1) / 2} & \mathbf{0}_{M-1 / 2} \\
\mathbf{0}_{1 \times(M-1) / 2} & \mathbf{0}_{1 \times(M-1) / 2} & 1 & \mathbf{0}_{1 \times(M-1) / 2} & \mathbf{0}_{1 \times(M-1) / 2} \\
\mathbf{0}_{(M-1) / 2} & \mathbf{0}_{(M-1) / 2} & \mathbf{0}_{(M-1) / 2 \times 1} & \mathbf{I}_{(M-1) / 2} & \mathbf{0}_{(M-1) / 2}
\end{array}\right]\left[\begin{array}{c}
\hat{\mathbf{x}}_{m} \\
M \\
\hat{\mathbf{x}}_{m+1}
\end{array}\right] \\
& =\frac{1}{2}\left[\begin{array}{c}
M \mathbf{u}+\mathbf{V q} \\
2 M \\
3 M \mathbf{u}-\mathbf{J V q}
\end{array}\right] .
\end{aligned}
$$



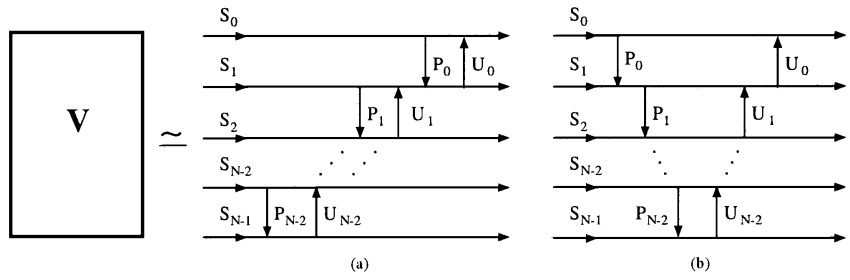

Fig. 3. Two lifting-based simplified models for the matrix V. (a) Type-III model. (b) Type-IV model.

\section{A. Type-III Fast Implementation}

In this simplified model, the $\lfloor M / 2\rfloor \times\lfloor M / 2\rfloor$ matrix $\mathbf{V}$ has three sets of parameters to be determined: $\lfloor M / 2\rfloor$ diagonal scaling factors $\left\{S_{i}\right\},\lfloor M / 2\rfloor-1$ predict lifting steps $\left\{P_{i}\right\}$, and $\lfloor M / 2\rfloor-1$ update lifting steps $\left\{U_{i}\right\}$. Those parameters should also satisfy the regularity conditions in Table I. We concentrate mainly on the 2-regular conditions since it is trivial to achieve the 1-regular condition.

Proposition 2: The synthesis scaling filter $f_{0}[n]$ constructed by the type-III pre-filter is 2-regular if and only if the design parameters in $\mathbf{V}$ satisfy the conditions shown at the bottom of the page.

Proof: Let $\mathbf{x}, \mathbf{y}$ be the input and output of the linear system $\mathbf{V}$, i.e., $\mathbf{y}=\mathbf{V x}$. If $\mathbf{V}$ satisfies the 2-regular constraint, then $\mathbf{x}=\mathbf{q}$ and $\mathbf{y}=M \mathbf{u}$.

For $i=0, \mathbf{y}_{0}=S_{0} \mathbf{x}_{0}+U_{0} \mathbf{y}_{1} \Longrightarrow M=S_{0}+M U_{0}$.

For $1 \leq i \leq\lfloor M / 2\rfloor-2, \mathbf{y}_{i}=S_{i} \mathbf{x}_{i}+P_{i-1} S_{i-1} \mathbf{x}_{i-1}+$ $U_{i} \mathbf{y}_{i+1} \Longrightarrow M=S_{i}(2 i+1)+P_{i-1} S_{i-1}(2 i-1)+U_{i} M$.

For $i=\lfloor M / 2\rfloor-1, \mathbf{y}_{i}=S_{i} \mathbf{x}_{i}+P_{i-1} S_{i-1} \mathbf{x}_{i-1} \Longrightarrow M=$ $(M-1) S_{i}+(M-3) S_{i-1} P_{i-1}$.

\section{B. Type-IV Fast Implementation}

Although the lifting structure of $\mathbf{V}$ in the type-III and -IV pre-/post-filters are different, they both have the same degree of freedom. If the parameters satisfy the 2-regular condition in Theorem 4, then $\mathbf{V}$ will produce a $\{1,2\}$-regular system. Following the same derivation as in Proposition 2, we can obtain the following proposition.

Proposition 3: The synthesis scaling filter $f_{0}[n]$ constructed by the type-IV pre-filter is 2-regular if and only if

$$
\begin{array}{r}
S_{0}+M U_{0}=M \\
(2 i+1) S_{i}+M\left(1-U_{i-1}\right) P_{i-1}+M U_{i}=M \\
\text { for } i=1,2, \ldots,\left\lfloor\frac{M}{2}\right\rfloor-2 \\
(M-1) S_{\lfloor M / 2\rfloor-1}+M\left(1-U_{\lfloor M / 2\rfloor-2}\right) P_{\lfloor M / 2\rfloor-2}=M .
\end{array}
$$

\section{Integer/Rational Implementation}

If the DCT is replaced by its integer approximations such as the binDCT [11], then $\mathbf{V}$ dictates the rational or integer property of the entire system since every other component already has integer coefficients. If all lifting steps as well as the diagonal scaling factors in Fig. 3 are chosen to be rational, then we have a complete system (both analysis and synthesis) with rational coefficients. Moreover, if these parameters are chosen to be dyadic, then we have an integer-coefficient analysis bank. The key to achieving integer-coefficient synthesis bank lies in the inversion of the diagonal scaling factors $S_{i}$. Table II tabulates several examples of parameter sets $\left\{P_{i}, U_{i}, S_{i}\right\}$ of the type-III and -IV dyadic rational $M$-band wavelets. All listed coding gains are computed with an $\operatorname{AR}(1)$ signal model $\rho=0.95$. Many more design examples can be found in Section V.

\section{DESIGN EXAMPLES}

\section{A. Design Process}

This section presents various filterbank design examples via regular-constrained pre-/post-filtering and the block DCT framework. We pay most attention to fast implementations and rational-coefficient $M$-band systems. All designs are obtained from the framework portrayed in Fig. 2 and the propositions presented in previous sections. Generally speaking, a regular-constrained pre-/post-filter has $\lfloor M / 2\rfloor \times(\lfloor M / 2\rfloor-1)$ free parameters, whereas an unconstrained solution has $(\lfloor M / 2\rfloor)^{2}$ free parameters. The number of parameters can be reduced to $3\lfloor M / 2\rfloor-2$ when $\mathbf{V}$ is modeled by the lifting structure in Section IV. In each design example, we convert the regular-constrained optimization problem into an unconstrained one by properly choosing regularity constraints developed in previous sections. The unconstrained optimization algorithm used to obtain all design examples in this paper is the Nelder-Mead simplex search available in Matlab Optimization Toolbox.

Since regularity has been robustly built into the pre-/postfilter's structure, we only optimize for one criterion in the design process - transform coding gain [12]-[14]:

$$
G_{T C}=10 \log _{10}\left(\frac{\frac{1}{N} \sum_{i=0}^{N-1} \sigma_{i}^{2}}{\left(\prod_{i=0}^{N-1} \sigma_{i}^{2} f_{i}^{2}\right)^{1 / N}}\right)
$$

where $\sigma_{i}^{2}$ is the variance of the $i$ th subband, and $f_{i}^{2}$ is the $\mathcal{L}^{2}$ norm of the $i$ th synthesis filter. When the transform is orthogonal, the synthesis scalings $f_{i}^{2}$ drop out of the coding gain equation. The input signal is assumed to be an $A R(1)$ model with autocorrelation coefficient $\rho=0.95$ throughout.

$$
\begin{gathered}
S_{0}+M U_{0}=M \\
(2 i+1) S_{i}+M U_{i}+(2 i-1) S_{i-1} P_{i-1}=M \text { for } i=1,2, \ldots \\
\left\lfloor\frac{M}{2}\right\rfloor-2 \\
(M-1) S_{\lfloor M / 2\rfloor-1}+(M-3) S_{\lfloor M / 2\rfloor-2} P_{\lfloor M / 2\rfloor-2}=M .
\end{gathered}
$$


TABLE II

EXAMPLES OF $M$-BAND WAVELET WITH RATIONAL/INTEGER PARAMETERS

\begin{tabular}{cc|cccc|cccccc|c}
\hline Size & Type & $S_{0}$ & $S_{1}$ & $S_{2}$ & $S_{3}$ & $P_{0}$ & $U_{0}$ & $P_{1}$ & $U_{1}$ & $P_{2}$ & $U_{2}$ & $C_{g}(A)(\mathrm{dB})$ \\
\hline \hline 4-band 8-tap & III, IV & $5 / 4$ & $23 / 16$ & - & - & $-1 / 4$ & $11 / 16$ & - & - & - & - & 8.533 \\
4-band 8-tap & III, IV & 2 & $3 / 2$ & - & - & $-1 / 4$ & $1 / 2$ & - & - & - & - & 8.266 \\
\hline 8-band 16-tap & IV & $3 / 2$ & $19 / 16$ & $21 / 16$ & $5 / 4$ & $-3 / 8$ & $13 / 16$ & $-3 / 16$ & $5 / 8$ & $-1 / 8$ & $1 / 4$ & 9.4898 \\
8-band 16-tap & IV & $3 / 2$ & $19 / 16$ & $9 / 8$ & $17 / 16$ & $-3 / 8$ & $13 / 16$ & $-3 / 8$ & $5 / 8$ & $1 / 8$ & $7 / 16$ & 9.4433 \\
\hline
\end{tabular}

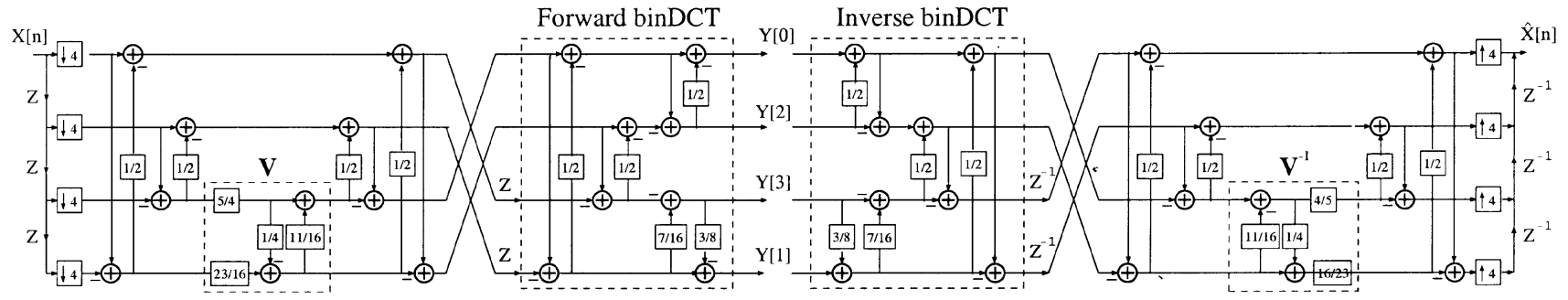

Fig. 4. Four-band 8-tap wavelet with dyadic and rational coefficients.

TABLE III

CoEfFicients of ANALYsis Filters IN the 4-BAND Design EXAMPLE 1

\begin{tabular}{l|rrrrrrrr}
\hline$h_{0}$ & $-365 / 256$ & $71 / 256$ & $441 / 256$ & $877 / 256$ & $877 / 256$ & $441 / 256$ & $71 / 256$ & $-365 / 256$ \\
\hline$h_{1}$ & $-983 / 1024$ & $133 / 1024$ & $1403 / 1024$ & $2007 / 1024$ & $-2007 / 1024$ & $-1403 / 1024$ & $-133 / 1024$ & $983 / 1024$ \\
\hline$h_{2}$ & $-141 / 512$ & $-89 / 512$ & $601 / 512$ & $-371 / 512$ & $-371 / 512$ & $601 / 512$ & $-89 / 512$ & $-141 / 512$ \\
\hline$h_{3}$ & $-29 / 512$ & $-169 / 512$ & $681 / 512$ & $-995 / 512$ & $995 / 512$ & $-681 / 512$ & $169 / 512$ & $29 / 512$ \\
\hline
\end{tabular}

\section{B. $\{1,2\}$-Regular 4-Band 8-Tap Filterbank}

Fig. 4 presents a 4-band 8-tap $\{1,2\}$-regular system with rational-coefficient pre-/post-filtering and the binDCT [11] as the block transform. The parameters used in constructing $\mathbf{V}$ are tabulated in the second row of Table II. The coefficients of the analysis filters $h_{i}[n]$ are listed in Table III. Because of the inversion of the diagonal scalars in the post-filter, the coefficients of the synthesis filters are rational instead of dyadic. Generally, it is difficult to make both banks dyadic while maintain good coding gain (the diagonal scaling factor $S_{i}$ has to be pure powers of two). The normalized frequency responses of the filters are shown in Fig. 5. The smoothness in the synthesis bank is evident from the synthesis scaling and wavelet function.

\section{C. $\{1,2\}$-Regular 8-Band 16-Tap Filterbank}

Fig. 6 presents an 8-band 16-tap \{1,2\}-regular design example under the fast type-III regular constraints in Proposition 2. The parameters used in the construction of $V$ are listed in the fourth row of Table II. Fig. 6 also shows the zero distribution of the analysis and the synthesis scaling filter, confirming that synthesis scaling filter has exactly three zeros at $z=-1$ and two zeros at all other aliasing frequencies. This is consistent with the fact that the synthesis scaling filter has the form $F_{0}(z)=\left[\left(1+z^{-1}+\cdots+z^{-7}\right) / 8\right]^{2}\left(a+a z^{-1}\right)$, which is the definition of an 8-band 2-regular scaling filter.

\section{D. $\{1,2\}$-Regular 16-Band 32-Tap Filterbank}

Systems with large number of channels can be designed as easily using the general constraint $\mathbf{V q}=M \mathbf{u}$. Fig. 7 shows a 16-band $\{1,2\}$-regular design example whose coding gain is $9.9364 \mathrm{~dB}$.

\section{E. $\{1,2\}$-Regular Odd-Band Filterbank}

We complete the section with two regular odd-band design examples. Fig. 8 shows a biorthogonal 5-band 9-tap filterbank constructed via the 5-point DCT and 4-point pre-/post-filtering. The 3-band rational-coefficient design example whose detailed lattice structure is illustrated in Fig. 9 is constructed from a 3-point binDCT and the 2-point pre-filter with $\mathbf{V}=[3]$.

\section{OBSERVATION}

\section{A. Pre- and Post-filtering Effects}

It is interesting to observe how pre- and post-filtering modify the signals in time domain. In Fig. 10, the solid line indicates the original signal, and the dotted line indicates the signal after pre-filtering. We can clearly observe the difference between the pre-filtering effects from a regular-constrained $\mathbf{V}$ and an orthogonal closed-form, yet nonregular, $\mathbf{V}$ suggested in [3]: $\mathbf{V}=$ $\mathbf{J C}_{M / 2}^{I I^{T}} \mathbf{C}_{M / 2}^{I V} \mathbf{J}$. The 2-regular constrained matrix $\mathbf{V}$ maps the ramp into piece-wise constant sequences of size $M$, whereas the unconstrained matrix $\mathbf{V}$ does not, confirming our previous results in Lemmas 1 and 2. The advantage of regular pre-/post-filtering is clear-after discarding all AC DCT coefficients, we can still reconstruct the ramp signal perfectly (except at the two boundaries) with the $\{1,2\}$-regular post-filter, whereas the $\{1,1\}$-regular post-filter still exhibits a fair amount of blocking artifacts. In any case, pre-/post-filtering clearly improve the traditional block DCT, which yields a perfect stair-case in this toy experiment.

Fig. 11 demonstrates the pre-filtering effect on the $256 \times 256$ gray-scale Slope image. The pre-filter tries to flatten each input block to the DCT as much as possible, hence improving the coding efficiency. Since interblock correlation has been 

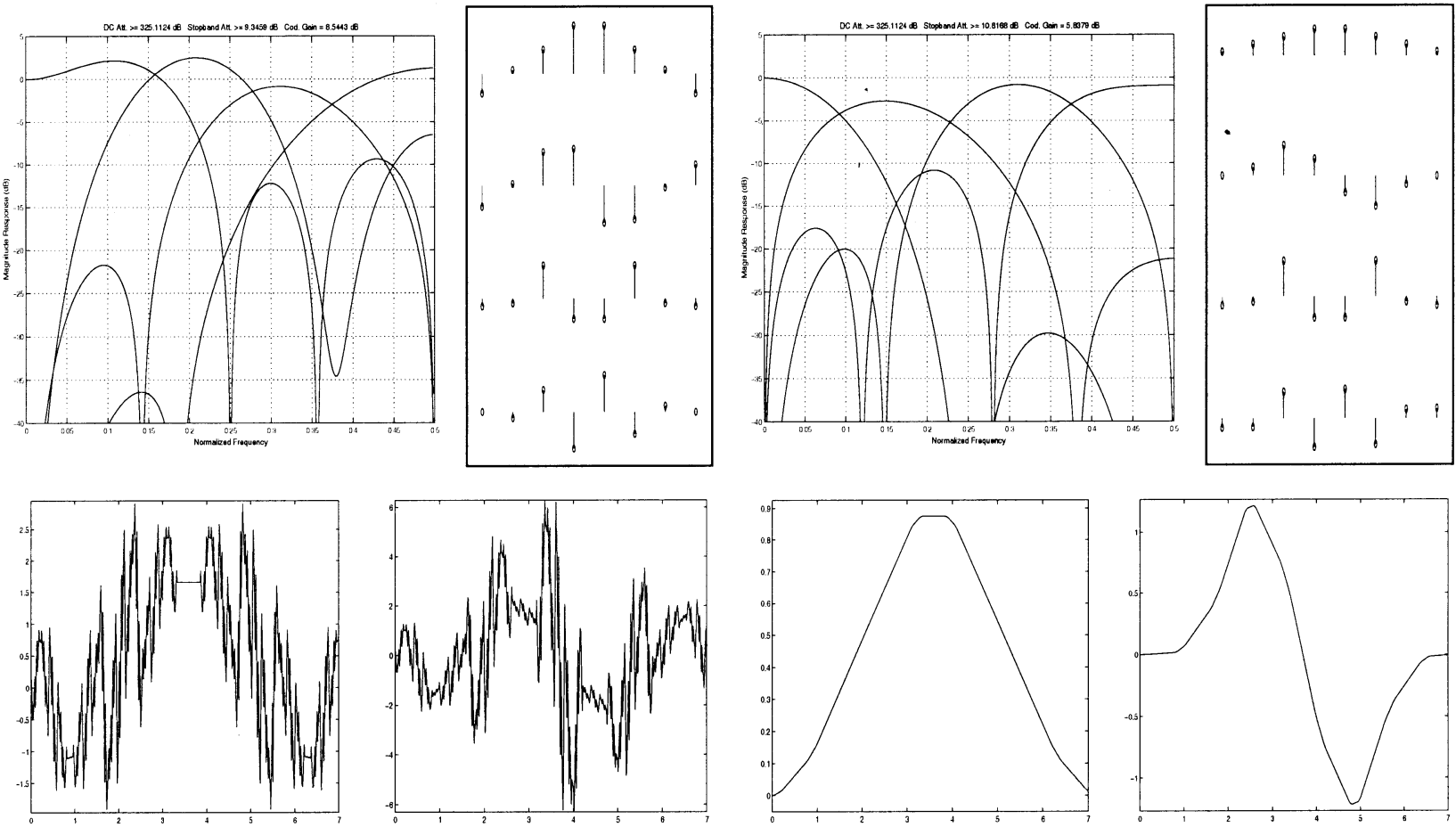

Fig. 5. Design example 1: $\{1,2\}$ regular 4-band 8-tap filterbank with 4-point binDCT. Top row from left to right: normalized frequency and time responses of the analysis filters (coding gain: $8.544 \mathrm{~dB}$ ), normalized frequency and time responses of the synthesis filters. Bottom row from left to right: analysis scaling function $\phi^{a}(t)$, first analysis wavelet function $\psi_{1}^{a}(t)$, synthesis scaling function $\phi^{s}(t)$, and first synthesis wavelet function $\psi_{1}^{s}(t)$.
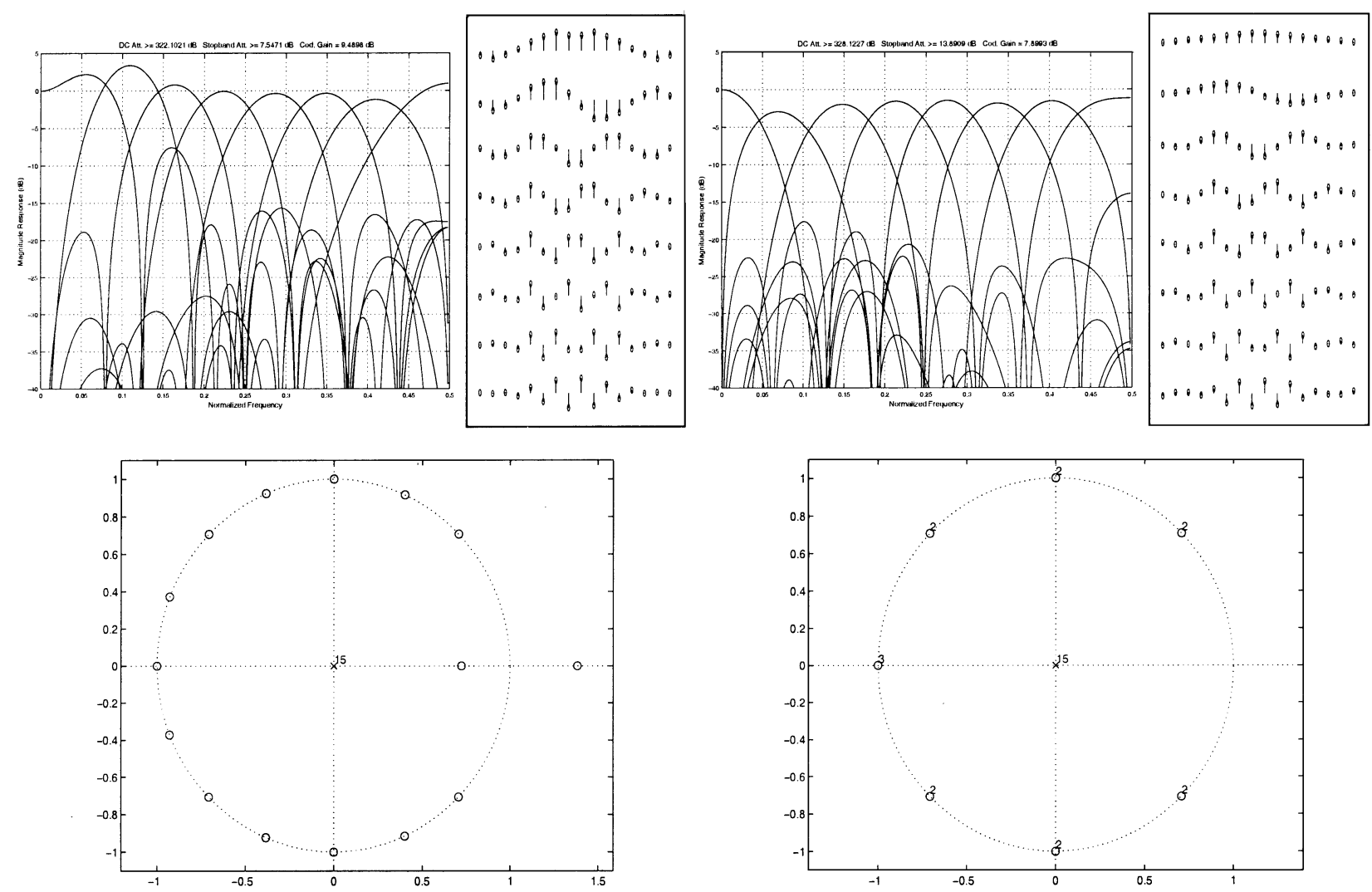

Fig. 6. Design example 2: $\{1,2\}$ regular 8-band 16-tap filterbank. Top row from left to right: normalized frequency and time responses of the analysis filters (coding gain: $9.4898 \mathrm{~dB}$ ), normalized frequency and time responses of the synthesis filters. Bottom row from left to right: zeros of the analysis scaling filter $h_{0}$ and zeros of the synthesis scaling filter $f_{0}$.

extracted in this process, the pre-filtered image becomes very blocky. For gradient-type signals, our regular-constrained pre-filter perfectly flattens each DCT input block, turning the input image into a chess-board. 

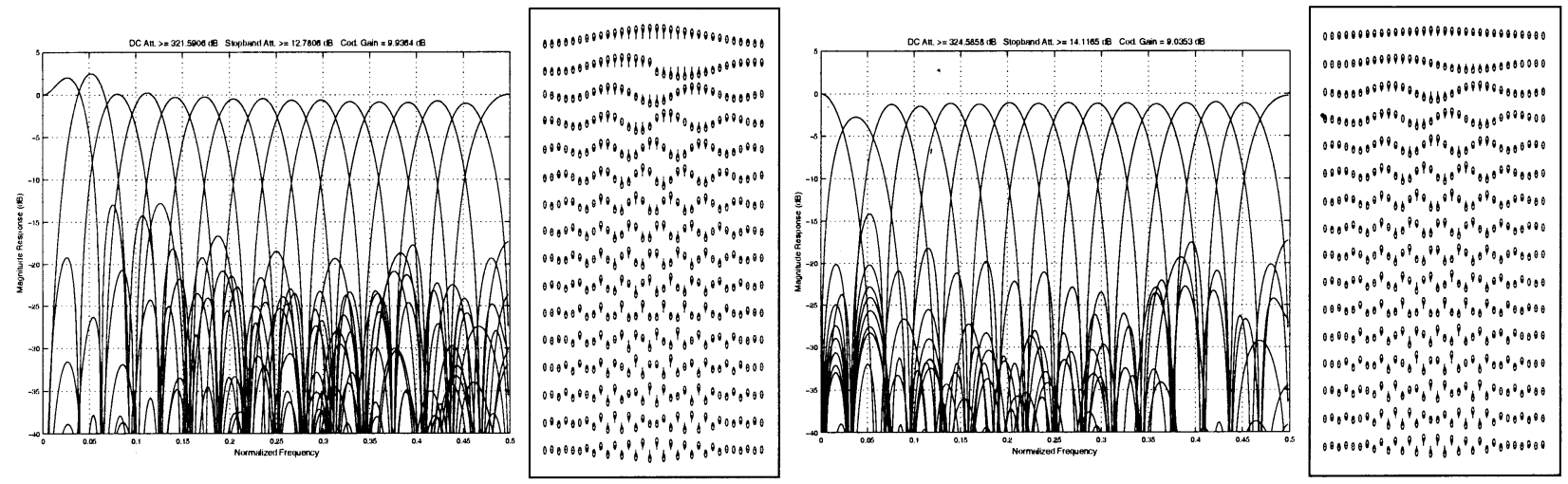

Fig. 7. Design Example 3: $\{1,2\}$ regular 16-band 32-tap filterbank. Top row from left to right: normalized frequency and time responses of the analysis filters (coding gain: $9.9364 \mathrm{~dB}$ ), normalized frequency and time responses of the synthesis filters.
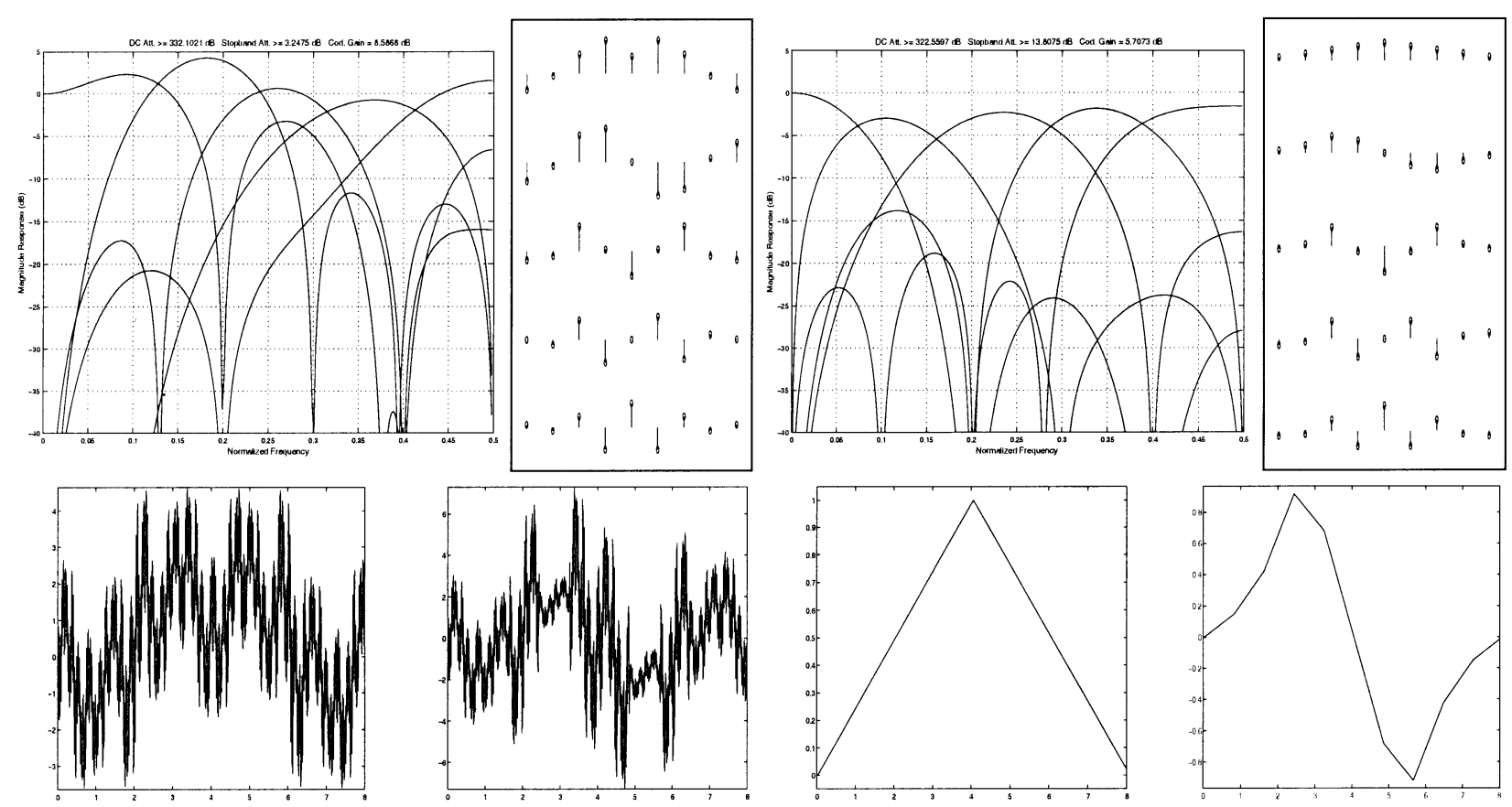

Fig. 8. Design example 4: $\{1,2\}$-regular 5-band 9-tap filterbank. Top row from left to right: normalized frequency and time responses of the analysis filters (coding gain: $8.5868 \mathrm{~dB}$ ), normalized frequency and time responses of the synthesis filters. Bottom row, from left to right: analysis scaling function $\phi^{a}(t)$, first analysis wavelet function $\psi_{1}^{a}(t)$, synthesis scaling function $\phi^{s}(t)$, and first synthesis wavelet function $\psi_{1}^{s}(t)$.

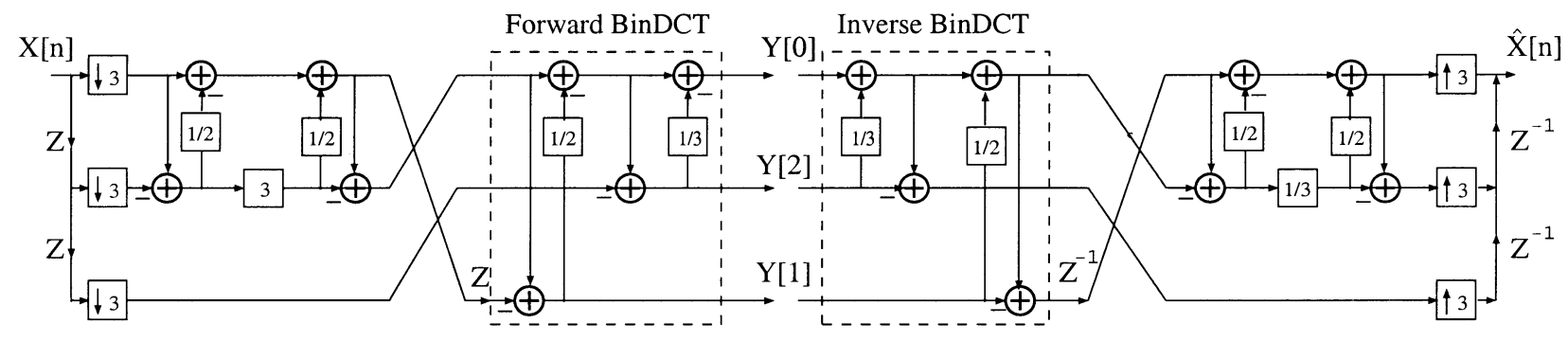

Fig. 9. Detailed lattice structure of a regular 3-band 5-tap filterbank with rational coefficients. 

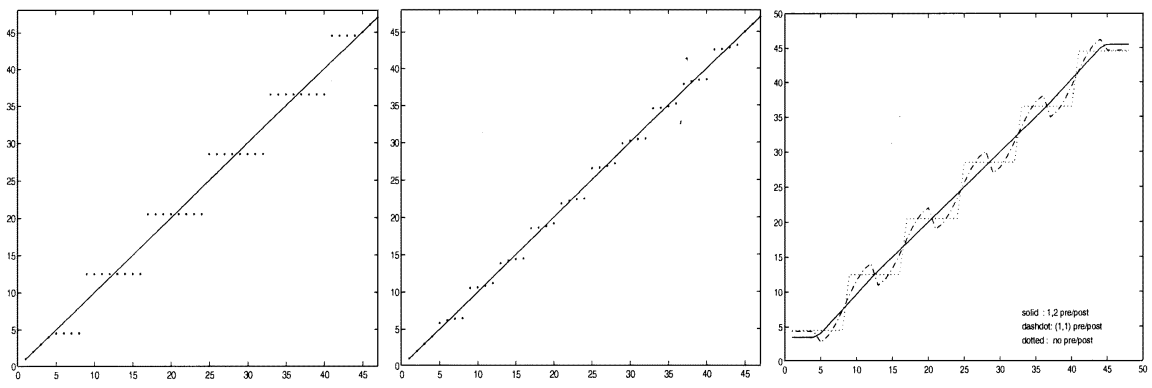

Fig. 10. Comparison between $\{1,2\}$-regular and $\{1,1\}$-regular pre- and post-filtering in the processing of a ramp signal. From left to right: pre-processing effect of a $\{1,2\}$-regular pre-filter and a $\{1,1\}$-regular pre-filter; reconstructed ramps from DC coefficients only.

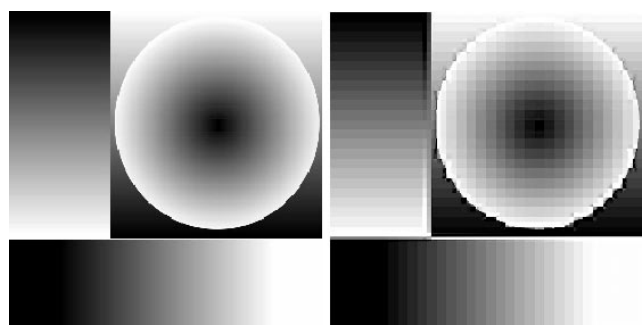

Fig. 11. Effects of $\{1,2\}$-regular preprocessing. Left: original Slope image. Right: after $\{1,2\}$-regular pre-filtering.
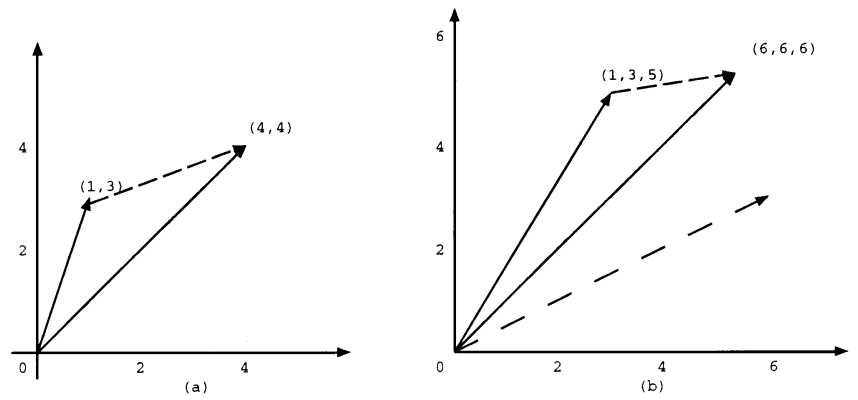

Fig. 12. Geometric interpretation of the regularity-constrained mapping operator V . (a) Two-dimensional mapping for block DCT of size 4. (b) Threedimensional mapping for block DCT of size 6.

\section{B. Geometric Interpretation}

The 2-regular constraint also has interesting geometric meaning. For $\mathbf{y}=\mathbf{V} \mathbf{x}$, we have $\|\mathbf{x}\|^{2}=\mathbf{x}^{T} \mathbf{x}$ and $\|\mathbf{y}\|^{2}=\mathbf{y}^{T} \mathbf{y}=\mathbf{x}^{T} \mathbf{V}^{T} \mathbf{V} \mathbf{x}$. If $\mathbf{V}$ is orthogonal, then $\|\mathbf{y}\|=\|\mathbf{x}\|$. In our 2-regular constraint, we have $\mathbf{x}=\mathbf{q}$ and $\mathbf{y}=M \mathbf{u}$. Since the norm of $\mathbf{q}$ is less than the norm of $M \mathbf{u}, \mathbf{V}$ cannot be an orthogonal matrix. In other words, we need a nonorthogonal matrix $\mathbf{V}$ to compensate for the norm of the ramp q. This means that only one of the following can hold: i) orthogonality and ii) 2-regularity. For example, when the block size is $4, \mathbf{V}$ should map vector $\left[\begin{array}{ll}1 & 3\end{array}\right]^{T}$ of norm $\sqrt{10}$ into vector $\left[\begin{array}{ll}4 & 4\end{array}\right]^{T}$ of norm $\sqrt{32}$. For a block size of $6, \mathbf{V}$ should map $\left[\begin{array}{lll}1 & 3 & 5\end{array}\right]^{T}$, which has norm $\sqrt{35}$ into $\left[\begin{array}{lll}6 & 6 & 6\end{array}\right]^{T}$, which has norm $\sqrt{108}$. The geometric interpretation is illustrated in Fig. 12. It is quite obvious that $\mathbf{V}$ cannot be an orthogonal projection operator in this case. This geometric interpretation offers an alternate intuitive proof that orthogonality and 2-regularity are mutually exclusive when only one stage of pre-/post-filtering is involved.
TABLE IV

COMPARISON OF OBJECTIVE CODING RESULTS (PSNR IN DECIBELS)

\begin{tabular}{|c|c|c|c|c|}
\hline Comp.Ratio & DCT & with pre/post & DCT & with pre/post \\
\hline & \multicolumn{2}{|c|}{ Goldhill $(512 \times 512)$} & \multicolumn{2}{|c|}{ Barbara $(512 \times 512)$} \\
\hline $1: 8$ & 34.36 & 34.64 & 33.05 & 34.11 \\
\hline $1: 16$ & 31.45 & 31.72 & 27.78 & 29.06 \\
\hline $1: 32$ & 28.33 & 28.85 & 24.34 & 25.00 \\
\hline \multirow[t]{2}{*}{$1: 64$} & 24.07 & 24.50 & - & 19.20 \\
\hline & \multicolumn{2}{|c|}{ Woman $(2048 \times 2560)$} & \multicolumn{2}{|c|}{ Bike $(2048 \times 2560)$} \\
\hline $1: 8$ & $\mathbf{3 4 . 4 4}$ & 34.19 & 34.17 & 33.72 \\
\hline $1: 16$ & 30.55 & $\overline{30.67}$ & 30.13 & 29.99 \\
\hline $1: 32$ & 27.08 & 27.17 & 26.29 & 26.25 \\
\hline \multirow[t]{2}{*}{$1: 64$} & 22.75 & 21.32 & - & 20.25 \\
\hline & \multicolumn{2}{|c|}{ Cafe $(2048 \times 2560)$} & \multicolumn{2}{|c|}{ Boat $(512 \times 512)$} \\
\hline $1: 8$ & 28.52 & 28.50 & 36.46 & 36.60 \\
\hline $1: 16$ & 24.36 & 24.55 & 32.25 & 32.67 \\
\hline $1: 32$ & 20.73 & 21.00 & 28.13 & 28.58 \\
\hline $1: 64$ & - & 15.59 & 23.66 & 23.96 \\
\hline
\end{tabular}

\section{C. $\{1,3\}$-Regular Biorthogonal Spline Wavelet}

When setting $M=2$ and $\mathbf{V}=$ [2], the pre-/post-filtering framework generates the following polyphase matrix:

$$
\begin{aligned}
\mathbf{E}(z)= & \frac{1}{\sqrt{2}}\left[\begin{array}{cc}
1 & 1 \\
1 & -1
\end{array}\right]\left[\begin{array}{ll}
0 & 1 \\
z & 0
\end{array}\right] \\
& \times \frac{1}{2}\left[\begin{array}{cc}
1 & 1 \\
1 & -1
\end{array}\right]\left[\begin{array}{cc}
1 & 0 \\
0 & 2
\end{array}\right]\left[\begin{array}{cc}
1 & 1 \\
1 & -1
\end{array}\right] \\
= & \frac{1}{\sqrt{2}}\left[\begin{array}{cc}
1 & 1 \\
1 & -1
\end{array}\right]\left[\begin{array}{ll}
0 & 1 \\
z & 0
\end{array}\right] \\
& \times \frac{1}{2}\left[\begin{array}{cc}
3 & -1 \\
-1 & 3
\end{array}\right] \\
= & \frac{\sqrt{2}}{4}\left[\begin{array}{cc}
-1+3 z & 3-z \\
-1-3 z & 3+z
\end{array}\right]
\end{aligned}
$$

which yields the scaled versions of the $4 / 4$ biorthogonal quadratic spline scaling filters

$$
\begin{aligned}
H_{0}(z) & =-\frac{1}{4}+\frac{3}{4} z+\frac{3}{4} z^{2}-\frac{1}{4} z^{3} \\
& =\left(\frac{1+z}{2}\right)\left(\frac{-1+4 z-z^{2}}{2}\right) \\
F_{0}(z) & =\frac{1}{4} z^{-3}+\frac{3}{4} z^{-2}+\frac{3}{4} z^{-1}+\frac{1}{4}=2\left(\frac{1+z^{-1}}{2}\right)^{3} .
\end{aligned}
$$

The synthesis bank has three vanishing moments; however, the analysis has only one. This is the single trivial example that $\{1,3\}$-regularity can be achieved with filter length $2 M$. 


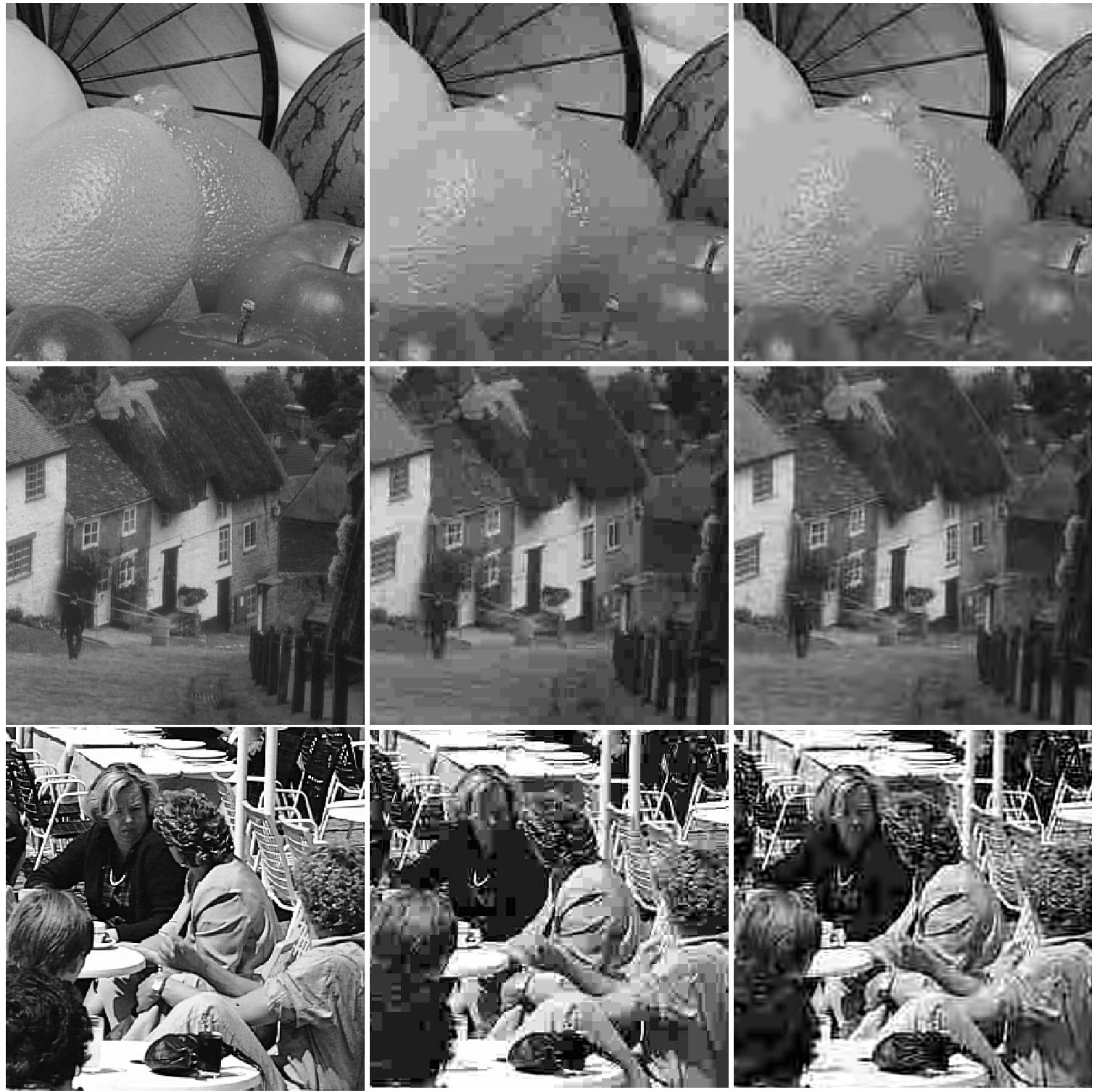

Fig. 13. Image portions at $0.25 \mathrm{~b}$ /pixel $(3 \times 300)$. From left to right: original image; coded by JPEG; coded by JPEG with the addition of the $\{1,2\}$-regular 8 -point pre-/post-filter. From top to bottom: bike at $(1656,1661)$, goldhill at $(211211)$, and cafe at $(950,1620)$.

\section{D. $\{1,2\}$-Regular 3-Band Wavelet}

In the case where $M=3$, the degenerated $\{1,2\}$-regular constraint requires $\mathbf{V}[1]=[3]$. Hence, by setting $\mathbf{V}=[3]$, the resulting pre-filter is

$$
\begin{aligned}
\mathbf{P} & =\frac{1}{2}\left[\begin{array}{cc}
1 & 1 \\
1 & -1
\end{array}\right]\left[\begin{array}{ll}
1 & 0 \\
0 & 3
\end{array}\right]\left[\begin{array}{cc}
1 & 1 \\
1 & -1
\end{array}\right] \\
& =\left[\begin{array}{cc}
2 & -1 \\
-1 & 2
\end{array}\right]
\end{aligned}
$$

which leads to the following polyphase matrix:

$$
\mathbf{E}(z)=\left[\begin{array}{ccc}
\frac{1}{\sqrt{3}} & \frac{1}{\sqrt{3}} & \frac{1}{\sqrt{3}} \\
\frac{1}{\sqrt{2}} & 0 & -\frac{1}{\sqrt{2}} \\
\frac{1}{\sqrt{6}} & -\frac{2}{\sqrt{6}} & \frac{1}{\sqrt{6}}
\end{array}\right]\left[\begin{array}{lll}
0 & 1 & 0 \\
0 & 0 & 1 \\
z & 0 & 0
\end{array}\right]\left[\begin{array}{ccc}
2 & -1 & 0 \\
-1 & 2 & 0 \\
0 & 0 & 1
\end{array}\right]
$$

$$
=\left[\begin{array}{ccc}
\frac{1}{\sqrt{3}}(-1+2 z) & \frac{1}{\sqrt{3}}(2-z) & \frac{1}{\sqrt{3}} \\
\frac{1}{\sqrt{2}}(-1-2 z) & \frac{1}{\sqrt{2}}(2+z) & 0 \\
\frac{1}{\sqrt{6}}(-1+2 z) & \frac{1}{\sqrt{6}}(2-z) & -\frac{2}{\sqrt{6}}
\end{array}\right] .
$$

The two corresponding analysis and synthesis scaling filters are

$$
\begin{aligned}
H_{0}(z) & =\frac{1}{3}\left(-1+2 z+z^{2}+2 z^{3}-z^{4}\right) \\
& =-\left(\frac{1+z+z^{2}}{3}\right)\left(1-3 z+z^{2}\right) \\
F_{0}(z) & =\frac{1}{9}\left(1+2 z^{-1}+3 z^{-2}+2 z^{-3}+z^{-4}\right) \\
& =\left(\frac{1+z^{-1}+z^{-2}}{3}\right)^{2} .
\end{aligned}
$$


The synthesis scaling filter has exactly two degrees of regularity and generates a triangular scaling (first-degree spline) filter.

\section{IMAGE CODING EXPERIMENT}

This section presents a simple image coding experiment, illustrating the potential application of our proposed regular pre-/post-filters. The coder chosen is the Joint Photographic Experts Group's baseline JPEG coder. The test images are standard 8-bit gray-scale: $512 \times 512$ Barbara, Goldhill, and Boat; $2048 \times 2560$ Bike, Cafe, and Woman.

Pre- and post-filtering modules are added outside the standard JPEG coding framework. The pre-/post-filter chosen for the experiment is the $\{1,2\}$-regular 8-point pre-/post-filter with

$$
\mathbf{V}=\left[\begin{array}{cccc}
0.9454 & 0.7917 & 0.4207 & 0.3680 \\
-0.5654 & 0.8863 & 0.6731 & 0.3630 \\
0.1118 & -0.3891 & 1.1034 & 0.5055 \\
-0.0312 & 0.0033 & -0.1386 & 1.2449
\end{array}\right]
$$

Pre-/post-filtering in 2-D is implemented in separable fashion. On the encoder side, since the preprocessing enlarges the dynamic range of the data samples, we have to make sure that the input sample value is within the 8-bit range [0,255], as most JPEG implementations require. Hence, pre-filtered samples are first shifted so that all input samples are non-negative. Then, these samples are prequantized to fit into the 8-bit range as the DCT in JPEG expects. Every option is set to default, except the quality factor, which is adjusted to control compression ratios. On the decoder side, JPEG-decoded samples are first dequantized and shifted to restore the original dynamic range and then fed into the post-filtering module.

From Table IV, it can be observed that the proposed pre-/postfilter helps JPEG improve PSNR in most cases. Several reconstructed image portions from various test images at 1:32 compression are depicted in Fig. 13. Subjectively, the combination of pre-/post-filtering and the block DCT is usually superior at preserving edges and textures while mitigating blocking artifacts. Without the $[0,255]$ range adjustment and with advanced context based coding, pre-/post-filtering has been shown to improve coding performance much more significantly [15], [16].

\section{CONCLUSION}

In this paper, we present the design and fast implementation of regularity constrained pre and post-filters for block-based DCT systems. We show that simple pre-and post-filtering modules added to the current block-based DCT infrastructure can help the block transform capture not only the constant signal but the ramp signal as well. Our proposed framework can be used to generate various fast symmetric $M$-band wavelets with up to two degrees of regularity. Coding experiments show that blocking is minimized, whereas edges and textures are better preserved compared with the traditional JPEG. Currently, we have only considered one stage of pre and post-processing. By adding more stages and extending the support of the pre-/postfilter, polynomials of higher orders can be captured, leading to smoother $M$-band scaling and wavelet functions. This is a topic for future research.

\section{REFERENCES}

[1] W. Pennebaker and J. Mitchell, JPEG Still Image Data Compression Standard. New York: Van Nostrand Reinhold, 1993.

[2] J. Mitchell, W. Pennebaker, C. Frogg, and D. LeGall, MPEG Video Compression Standard. New York: Chapman and Hall, 1996.

[3] T. D. Tran, "Lapped transform via time-domain pre- and post-processing," in Proc. Conf. Inform. Sci. Syst., Mar. 21, 2001, pp. 890-895.

[4] M. Vetterli and J. Kovacevic, Wavelets and Subband Coding. Englewood Cliffs, NJ: Prentice-Hall, 1995.

[5] P. Vaidyanathan, Multirate Systems and Filter Banks. Englewood Cliffs, NJ: Prentice-Hall, 1993.

[6] G. Strang and T. Nguyen, Wavelets and Filter Banks. Wellesley, MA: Wellesley-Cambridge Press, 1997.

[7] P. Steffen, P. N. Heller, R. A. Gopinath, and C. S. Burrus, "Theory of regular M-Band wavelet bases," IEEE Trans. Signal Processing, vol. 41, pp. 3497-3511, Dec. 1993.

[8] S. Oraintara, "Regular linear phase perfect reconstruction filter banks for image compression," Ph.D. dissertation, Boston Univ., Boston, MA, 2000.

[9] C. S. Burrus, R. A. Gopinath, and H. Guo, Introduction to Wavelets and Wavelet Transforms: A Primer. Englewood Cliffs, NJ: Prentice-Hall, 1998.

[10] T. D. Tran, J. Liang, and C. Tu, "Lapped transform via time-domain pre- and post-filtering," IEEE Trans. Signal Processing, vol. 51, pp. 1557-1571, June 2003

[11] J. Liang and T. D. Tran, "Fast multiplierless approximations of the DCT with the lifting scheme," IEEE Trans. Signal Processing, vol. 49, pp. 3032-3044, Dec. 2001.

[12] J. Katto and Y. Yasuda, "Performance evaluation of subband coding and optimization of its filter coefficients," in Proc. SPIE Visual Commun. Image Process., Boston, MA, Nov. 1991, pp. 95-106.

[13] H. S. Malvar, "Biorthogonal and nonuniform lapped transforms for transform coding with reduced blocking and ringing artifacts," IEEE Trans. Signal Processing, vol. 46, pp. 1043-1053, Apr. 1998.

[14] T. D. Tran, "M-channel linear phase perfect reconstruction filter bank with rational coefficients," IEEE Trans. Circuits Syst. I, vol. 49, pp. 914-927, July 2002.

[15] C. Tu and T. D. Tran, "Context based entropy coding of block transform coefficients for image compression," in Proc. SPIE Applicat. Digital Image Processing XXIV, San Diego, CA, Aug. 2001, pp. 377-389.

[16] _ - "Context based entropy coding of block transform coefficients for image compression," IEEE Trans. Image Processing, vol. 11, pp. 1271-1283, Nov. 2002.

[17] N. Ahmed, T. Natarajan, and K. R. Rao, "Discrete cosine transform," IEEE Trans. Comput., vol. C-23, pp. 90-93, Jan. 1974.

[18] O. Alkin and H. Caglar, "Design of efficient M-band coders with linearphase and perfect-reconstruction properties," IEEE Trans. Signal Processing, vol. 43, pp. 1579-1590, July 1995.

[19] W. Chen, C. Harrison, and S. Fralick, "A fast computational algorithm for the discrete cosine transform," IEEE Trans. Commun., vol. COM-25, pp. 1004-1011, 1977.

[20] T. D. Tran and T. Q. Nguyen, "A progressive transmission image coder using linear phase filter banks as block transforms," IEEE Trans. Image Processing, vol. 8, pp. 1493-1507, Nov. 1999.

[21] I. Daubechies and W. Sweldens, "Factoring wavelet transforms into lifting step,” J. Fourier Anal. Appl., vol. 4, pp. 247-269, 1998.

[22] R. Gopinath, J. Odegard, and C. Burrus, "Optimal wavelet representation of signals and the wavelet sampling theorem," IEEE Trans. Circuits Syst. II, vol. 41, pp. 262-277, Apr. 1994.

[23] W. Dai and T. D. Tran, "Regularity-constrained pre- and post-filtering for block-based coding and communication systems," in Proc. ICICS, Singapore, Oct. 2001.

[24] N. S. Jayant and P. Noll, Digital Coding of Waveforms. Englewood Cliffs, NJ: Prentice-Hall, 1984

[25] H. S. Malvar and D. H. Staelin, "The lot: Transform coding without blocking effects," IEEE Trans. Signal Processing, vol. 37, pp. 553-559, Apr. 1989.

[26] S. Oraintara, T. D. Tran, P. Heller, and T. Q. Nguyen, "Lattice structure for regular paraunitray linear phase filter banks and M-band orthogonal symmetric wavelets," IEEE Trans. Signal Processing, vol. 49, pp. 2659-2672, Nov. 2001.

[27] H. S. Malvar, Signal Processing With Lapped Transforms. Norwood, MA: Artech House, 1992.

[28] - "Fast progressive image coding without wavelets," in Proc. Data Compression Conf., Snowbird, UT, Mar. 2000, pp. 243-252. 
[29] R. A. Horn and C. R. Johnson, Matrix Analysis. Cambridge, U.K.: Cambridge Univ. Press, 1999.

[30] C. A. Segall and A. K. Katsaggelos, "Pre- and post-processing algorithms for compressed video enhancement," in Proc. Asilomar Conf. Signals Syst., Pacific Grove, CA, Oct. 2000, pp. 1369-1373.

[31] K. R. Rao and P. Yip, Discrete Cosine Transform: Algorithms, Advantages, Applications. New York: Academic, 1990.

[32] A. K. Soman, P. P. Vaidyanathan, and T. Q. Nguyen, "Linear phase paraunitary filter banks: Theory, factorizations and applications," IEEE Trans. Signal Processing, vol. 41, pp. 3480-3496, Dec. 1993.

[33] A. Said and W. A. Pearlman, "A new fast and efficient image codec based on set partitioning in hierarchical trees," IEEE Trans Circuits Syst. Video Technol., vol. 6, pp. 243-250, June 1996.

[34] J. Zhang and Z. Bao, "Property of vanishing moments of orthogonal M-band compactly supported interpolating scaling function," Electron. Lett., vol. 34, pp. 1917-1918, Oct. 1998.

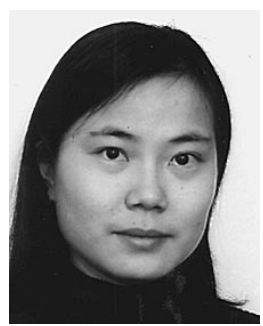

Wei Dai (S'01) received the B.E. degree from Xian Jiaotong University, Xian, China, in 1996 and the M.E. degree from Beijing University of Posts and Telecommunications, Beijing, China, in 1999. She is currently pursuing the Ph.D. degree at the Department of Electrical and Computer Engineering,The Johns Hopkins University, Baltimore, MD.

Her current research interests are in the area of multirate signal processing and wavelet-based signal processing and its applications.

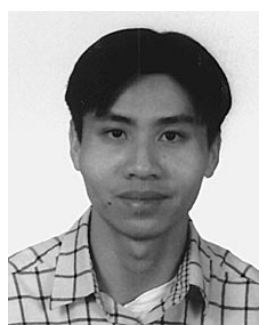

Trac D. Tran (S'94-M'98) received the B.S. and M.S. degrees from the Massachusetts Institute of Technology, Cambridge, in 1993 and 1994, respectively, and the Ph.D. degree from the University of Wisconsin, Madison, in 1998, all in electrical engineering. He joined the Department of Electrical and Computer Engineering, The Johns Hopkins University, Baltimore, MD, in July 1998 and is currently an Associate Professor. His research interests are in the field of digital signal processing, particularly in multirate systems, filterbanks, transforms, wavelets, and their applications in signal analysis, compression, processing, and communications. In the summer of 2002, he was an ASEE/ONR Summer Faculty Research Fellow at the Naval Air Warfare Center Weapons Division (NAWCWD), China Lake, CA.

Dr. Tran currently serves as Associate Editor of the IEEE TRANSACTIONS ON Signal Processing and the IEEE TRANSACTIONS ON IMAGE PROCESSING. He was the co-director (with Prof. J. L. Prince) of the 33rd Annual Conference on Information Sciences and Systems (CISS'99), Baltimore, MD, in March 1999. He received the NSF CAREER award in 2001. 